\title{
RhoA/Rock activation represents a new mechanism for inactivating Wnt/ $\beta$-catenin signaling in the aging-associated bone loss
}

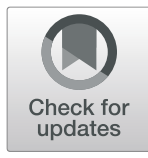

Wei Shi ${ }^{1,2+}$, Chengyun Xu ${ }^{1,3+}$, Ying Gong ${ }^{1}$, Jirong Wang ${ }^{1}$, Qianlei Ren ${ }^{4}$, Ziyi Yan ${ }^{1}$, Liu Mei ${ }^{1}$, Chao Tang ${ }^{1}$, Xing Ji ${ }^{1,5}$, Xinhua Hu' ${ }^{1}$, Meiyu Qv' ${ }^{1}$, Musaddique Hussain ${ }^{1}$, Ling-Hui Zeng ${ }^{4^{*}}$ and Ximei $\mathrm{Wu}^{1,3^{*}}$ (D)

\begin{abstract}
The Wnt/ $\beta$-catenin signaling pathway appears to be particularly important for bone homeostasis, whereas nuclear accumulation of $\beta$-catenin requires the activation of Rac1, a member of the Rho small GTPase family. The aim of the present study was to investigate the role of RhoA/Rho kinase (Rock)-mediated Wnt/ $\beta$-catenin signaling in the regulation of aging-associated bone loss. We find that Lrp5/6-dependent and Lrp5/6-independent RhoA/Rock activation by Wnt3a activates Jak1/2 to directly phosphorylate Gsk3 $\beta$ at Tyr216, resulting in Gsk3 $\beta$ activation and subsequent $\beta$-catenin destabilization. In line with these molecular events, RhoA loss- or gain-of-function in mouse embryonic limb bud ectoderms interacts genetically with $D k k 1$ gain-of-function to rescue the severe limb truncation phenotypes or to phenocopy the deletion of $\beta$-catenin, respectively. Likewise, RhoA loss-of-function in pre-osteoblasts robustly increases bone formation while gain-of-function decreases it. Importantly, high RhoA/Rock activity closely correlates with Jak and Gsk3 $\beta$ activities but inversely correlates with $\beta$-catenin signaling activity in bone marrow mesenchymal stromal cells from elderly male humans and mice, whereas systemic inhibition of Rock therefore activates the $\beta$-catenin signaling to antagonize aging-associated bone loss. Taken together, these results identify RhoA/Rock-dependent Gsk3 $\beta$ activation and subsequent $\beta$-catenin destabilization as a hitherto uncharacterized mechanism controlling limb outgrowth and bone homeostasis.
\end{abstract}

Keywords: RhoA, Rock, Wnt, $\beta$-Catenin, Limb bud, Bone

\section{Background}

Wnt ligands activate both $\beta$-catenin-dependent and $\beta$ catenin-independent signaling pathways and play important roles in embryonic development and tissue homeostasis (van Amerongen and Nusse 2009). During Wnt/ $\beta$-catenin signaling, the binding of Wnt to Frizzled (FZD) receptors and to the low-density lipoprotein receptor-related protein 5 or $6(\operatorname{Lrp} 5 / 6)$ activates

\footnotetext{
* Correspondence: zenglh@zucc.edu.cn; xiwu@zju.edu.cn

${ }^{+}$Wei Shi and Chengyun Xu contributed equally to this work.

${ }^{4}$ Department of Pharmacology, Zhejiang University City College, 51 Huzhou Street, Hangzhou 310015, China

'Department of Pharmacology, Zhejiang University School of Medicine, 866 Yuhangtang Road, Hangzhou 310058, China

Full list of author information is available at the end of the article
}

Dishevelled (Dvl) to stabilize cytosolic $\beta$-catenin. Upon its entry into the nucleus, $\beta$-catenin in turn activates the transcription of downstream target genes, including lymphoid enhancer-binding factor 1 (Lef1), cyclin D1 (C.D1), Dickkopf-1 (Dkk1), and Axin2 (Huelsken and Birchmeier 2001; Wu et al. 2008). The secreted proteins Dkk1 and sclerostin (Sost) then antagonize the pathway by interfering with Lrp5/6-Wnt interactions, thereby providing a negative feedback mechanism that limits the range of Wnt signaling (Bafico et al. 2001; Wu et al. 2008).

Wnt/ $\beta$-catenin signaling functions as a master regulator of limb development and bone formation; consequently, dysregulation of this pathway results in severe 
limb defects in mouse embryos and a failure to maintain bone mass in adult mice and humans (Barrow et al. 2003; Canalis et al. 2007; Karner and Long 2017). Notably, patients with osteoporosis or age-associated bone loss display a significantly low activity of $\mathrm{Wnt} / \beta$-catenin signaling in bone marrow mesenchymal stromal cells (BMMSCs), primarily due to the high circulating levels of Dkk1 and Sost (Coulson et al. 2017; Hampson et al. 2013). Targeting the Wnt/ $\beta$-catenin signaling therefore represents a promising therapeutic approach for treatment of osteoporosis or aging-associated bone loss (Jing et al. 2018; Manolagas and Almeida 2007).

Wnt signaling also occurs via two well-characterized $\beta$-catenin-independent pathways-the planar cell polarity (PCP) pathway and the $\mathrm{Wnt} / \mathrm{Ca}^{2+}$ pathway-and both pathways inhibit $\beta$-catenin-dependent Wnt signaling (Nemeth et al. 2007; Yuzugullu et al. 2009). The PCP pathway is initiated by Wnt binding to Frizzled receptors and leads to the downstream activation of Rho family small GTPases (Fanto et al. 2000; Strutt et al. 1997). This family of GTPases includes RhoA, Rac1, and Cdc42, which serve as molecular switches with multiple roles that involve cycling between active GTP-bound and inactive GDP-bound forms (Bishop and Hall 2000). We have previously reported that nuclear accumulation of $\beta$ catenin requires the activation of Rac1. Specifically, we have shown that activation of c-Jun $\mathrm{N}$-terminal kinase 2 (JNK2) by Rac1 results in phosphorylation of $\beta$-catenin on critical residues and promotes its nuclear translocation. We have also presented evidence that Rac1 interacts genetically with $\beta$-catenin and $D k k 1$ to control limb outgrowth in mouse embryos (Wu et al. 2008).

Despite this discovery of a role for Rho small GTPases in the $\beta$-catenin-dependent Wnt signaling pathway, the importance and biological relevance of RhoA in the regulation of $\mathrm{Wnt} / \mathrm{\beta}$-catenin signaling remain obscure. Here, we report that the regulation of aging-associated bone loss involves the destabilization of $\beta$-catenin by RhoA/Rho kinase (Rock) through activation of Janus kinase (Jak) and its direct phosphorylation of the Tyr216 residue of glycogen synthase kinase-3 $\beta$ (Gsk3 $\beta$ ).

\section{Methods}

\section{Antibodies, proteins, chemicals, and kits}

Antibodies against Jak1, Jak2, Gsk3 $\beta$, $\beta$-catenin, p-Ser9Gsk3 $\beta$, p-Ser552- $\beta$-catenin, $p$-Ser33/37/Thr41- $\beta$-catenin (p-Ser33- $\beta$-catenin), and non-p-Ser45- $\beta$-catenin (active $\beta$-catenin) were from Cell Signaling (Danvers, MA). Antibodies against p-Tyr1022-Jak1 and p-Tyr1007-Jak2 were purchased from Bioworld Technology (St. Louis Park, MN). Antibodies against Rock1, Rock2, Gsk3 $\beta$, glyceraldehyde-3-phosphate dehydrogenase (GAPDH), $\beta$-actin, and Lamin B were obtained from Santa Cruz Biotechnology (Santa Cruz, CA). Antibody against alkaline phosphatase (Alp), bone sialoprotein (Bsp), Lef1, Nestin, p-Ser1366-Rock2, and p-Tyr216-Gsk3 $\beta$ and recombinant human active Jak2 and Gsk3 $\beta$ were from Abcam (Cambridge, UK). The IRDye 680, 700, and $800 \mathrm{~s}$ antibodies were from LI-COR Bioscience (Lincoln, NE). Alexa555- and Alexa488-conjugated secondary antibodies were from Molecular Probes (Eugene, OR). Recombinant mouse active Wnt3a (rWnt3a), Wnt5a (rWnt5a), and Dkk1 (rDkk1) were from StemRD (Burlingame, CA). Y27632 and Fasudil were from Selleckchem (Huston, TX) and Pyridone 6 was from Medchem Express (Monmouth Junction, NJ).

\section{Mouse strains, treatments, and embryos}

Msx2-Cre, Col1-Cre (2.3 kb), CAT-dnRhoA ${ }^{+-}, \quad$ R26$D k k 1^{+/-}$, and $\beta$-catenin ${ }^{f / f}$ mouse strains were as reported (Sun et al. 2000; Kobayashi et al. 2004; Wu et al. 2008; Barrow et al. 2003). The $R h o A^{f / f}$ mouse strain was generated with CRISPR/Cas9- mediated genome-editing technique by Nanjing Biomedical Research Institute of Nanjing University (Nanjing, China) as described previously (Ma et al. 2014). The conditional caRhoA (G14V) knock-in mouse strain was generated by Cyagen Biosciences Inc. (Guangzhou, China) as described previously (Xu et al. 2019; Jackson et al. 2001). Mouse experiments were approved by the Zhejiang University Institutional Animal Care and Use Committee. Embryos were harvested for whole-mount skeleton preparation, whole-mount in situ hybridization, and limb bud sectioning and staining as previously described (Wu et al. 2008; Tu et al. 2007). Five-, 8-, and 10-monthold male Institute of Cancer Research (ICR) mice were purchased from Laboratory Animal Center of Zhejiang Province (Hangzhou, China). Two-, 5-, 8-, and 10-month-old male ICR mice were used for isolation of BMMSCs, and were also subjected to tibial sectioning. Eight-month-old male ICR mice were orally administrated with Fasudil once daily at 0 and $100 \mathrm{mg} / \mathrm{kg}$ for 2 months. Long bones were used for $\mu \mathrm{CT}$ examination or sectioning, whereas sera were used for ELISA assays of carboxyterminal collagen crosslinks (CTX) (CUSABIO, Wuhan, China), tartrate-resistant acid phosphatase (Trap) (Immunodiagnostic Systems, Scottsdale, AZ), osteocalcin (OCN) (Immutopics, San Clemente, CA), and procollagen type $1 \mathrm{~N}$-terminal propeptide (P1NP) (MyBioSource, San Diego, CA). We used $\mu \mathrm{CT}$ ( $\mu \mathrm{CT}$ 40, Scanco Medical) for three-dimensional reconstruction and quantification of bone parameters, and reconstructed each image from one hundred $16-\mu \mathrm{m}$ slices immediately below the growth plate, with a threshold of 200 (Gong et al. 2014; Hu et al. 2018).

\section{Isolation of human BMMSCs}

Bone marrows were harvested from four elderly men (74-, 78-, 82-, and 84-year-old) who suffered from femoral neck fracture and underwent the total hip 
replacement surgery and three young men (28-, 32-, 33year-old) who suffered from traumatic fracture and underwent the internal fixation surgery in the Department of Orthopeadic Surgery, Sir Run Run Shaw Hospital, Zhejiang University School of Medicine. The young men were hurt in accidents and had no osteoporosis or other diagnosed diseases. The elderly men were satisfied with the diagnosis of osteoporosis according to the commonly accepted WHO definitions and criteria, and had no other diagnosed diseases. Bone marrows were used for preparation of smears or isolation of BMMSCs by the Human Marrow Lymphocyte Medium Kit (Sangon Biotech, Shanghai, China). For quantification of bone marrow smear staining, phospho-ROCK2 $2^{+}$, p-Ser33- $\beta$-CATENIN ${ }^{+}$, active $\beta$-CATENIN ${ }^{+}, \mathrm{p}$-JAK $1 / 2^{+}$, or p-Tyr216-GSK3 $\beta^{+}$cells were raised from every 200 NESTIN $^{+}$cells.

\section{Isolation and culture of mouse BMMSCs}

Mouse BMMSCs were isolated by a MagCellect Mouse Mesenchymal Stem Cell Isolation Kit (R\&D Systems, Minneapolis, MN) as described previously (Gong et al. 2014). BMMSCs were cultured in the Roswell Park Memorial Institute (RPMI) medium supplemented with $10 \%$ heatinactivated fetal calf serum (FCS) (Invitrogen, Carlsbad, CA), $100 \mathrm{IU} / \mathrm{ml}$ penicillin, and $100 \mathrm{mg} / \mathrm{ml}$ streptomycin (Invitrogen). Culture medium was changed at day 3 to remove the nonadherent cells. Cells were grown for $3 \sim 4$ weeks until almost confluency. Adherent cells were then detached and replated using a 1:3 dilution until passage 2 . Subsequent passaging and seeding of the cells were performed at a density of 5000 cells $/ \mathrm{cm}^{2}$. BMMSCs were used for experiments after $4 \sim 7$ passages.

\section{Cell cultures}

Primary mouse calvarial osteoblasts (PMCOBs) were isolated from neonatal mice at postnatal day (P) $2 \sim 5$, and expanded and maintained as described previously (Gong et al. 2014; Hu et al. 2018; Chen et al., 2017). C3H10T1/ 2 cells, HEK293T cells, and L929 cells were obtained from the American Type Culture Collection (ATCC), authenticated using short tandem repeat (STR) profiling, and maintained as described previously (Gong et al. 2014; $\mathrm{Hu}$ et al. 2018; Chen et al., 2017). No cell line used in this study was found in the database of commonly misidentified cell lines that is maintained by ICLAC and NCBI Biosample. Cells were passaged for no more than 6 months and routinely assayed for mycoplasma contamination.

\section{Transfection, lentiviral infection, dual-luciferase and rho} activation assays

Constructs expressing the interest genes were cloned by using specific primers (Table S1), and gene-specific
siRNAs were synthesized by Sangon Biotech (Shanghai, China) (Table S2). Transient transfection and Lef1-luciferase reporter assay were performed in C3H10T1/2 cells by using Lipofectamine 2000 (Invitrogen) as described previously (Gong et al. 2014; Hu et al. 2018). Dualluciferase assay was performed according to the manufacturer's instructions (Promega, Madison, WI). The firefly luciferase activities were normalized to Renilla luciferase levels. Infection and preparation of lentiviruses expressing the interest genes or shRNAs were as described previously (Table S3) (Hu et al. 2018). Assays for RhoA, Rac1 and Cdc42 activation were performed by using Active GTPase Pull-Down and Detection Kits (Pierce Biotechnology, Rockford, IL) as described previously (Wu et al. 2008). Total RhoA, Rac1, and $\mathrm{Cdc} 42$ were used as internal controls for their active forms (GTP binding form).

\section{Western blotting, immunoprecipitation, and in vitro phosphorylation assays}

The cytosolic and nuclear fractions of cells were prepared as previously described (Wu et al. 2008; Gong et al. 2014). Immunoprecipitation and western blot analyses were performed using standard protocols as previously described (Wu et al. 2008). The in vitro kinase assay to assess the phosphorylation of GSK3 $\beta$ by JAK2 was performed as previously described (Wu et al. 2008). To validate JAK2 as a kinase phosphorylating GSK3 $\beta$ at Y216, recombinant human active JAK2 (Abcam, ab42621) and human GSK3 $\beta$ (Abcam, ab63193) were used in the in vitro kinase assay. $30 \mathrm{ng}$ of GSK3 $\beta$ was incubated with $50 \mu \mathrm{l}$ kinase assay buffer (Cell Signaling) in the presence or absence of $30 \mathrm{ng}$ active JAK2 and $2 \mathrm{mM} \mathrm{ATP}$ at $37^{\circ} \mathrm{C}$ for $30 \mathrm{~min}$. The reactive mixture was subjected to western analyses with antibodies recognizing p-Tyr216-GSK3 $\beta$, GSK3 $\beta$, and JAK2. For western, GAPDH or $\beta$-actin was used as the internal standard of total target proteins, and phosphorylated proteins were normalized to total proteins, respectively. Immunoreactive bands from duplicates or triplicates were quantified by ImageJ, and the mean intensity from first band was set to 1 .

\section{Osteoblast differentiation assay and quantitative RT-PCR}

C3H10T1/2 cells or PMCOBs were subjected to Alp activity and formation of mineralized nodule assays as previously described (Wu et al. 2008; Gong et al. 2014). Alp activity was expressed as nanomoles of pnitrophenol formed per minute per milligram of protein. For mineralization assays, cells were incubated in the regular growth medium containing $10 \%$ FCS, $50 \mathrm{mg} / \mathrm{ml}$ ascorbic acid, $100 \mathrm{nM}$ dexamethasone, and $10 \mu \mathrm{M} \beta$ glycerophosphate $(\beta-\mathrm{GP})$ for 21 days. Cells were stained with Alizarin-Red S (Sigma) and then extracted with 
$10 \%$ cetylpyridinium chloride (Sigma) for quantification as previously described (Chen et al., 2017). RNA was extracted from cultured cells or femurs by using TRIzol reagent (Takara Biotechnology). Messenger RNAs of Alp, Bsp, Runx2, Lef1, Cyclin D1, and Axin2 were assayed as previously described (Gong et al. 2014). GAPDH or $\beta$ actin was included as an internal control and the relative values of mRNA species of interest were calculated by the $2^{-\Delta \Delta \mathrm{Ct}}$ method.

\section{Histology, immunostaining, and dynamic bone histomorphometry}

Mice were euthanized by $\mathrm{CO}_{2}$ asphyxiation, and tissues were fixed in $4 \%$ paraformaldehyde followed by decalcification and processing. Selected bones were embedded and sectioned for H\&E staining, Trap staining, and immunostaining as described previously (Gong et al. 2014; $\mathrm{Hu}$ et al. 2018; Chen et al., 2017). The quantitative histomorphometry was performed using the Image $(\mathrm{NIH})$ and Osteomeasure Analysis System (Osteometrics, Decatur, GA) according to the recommendations of the Histomorphometry Nomenclature Committee of the American Society of Bone and Mineral Research (Dempster et al. 2013). Every 200 Nestin $^{+}$cells were manually scored as either positive or negative staining for phospho-Rock2, pJak1/2, p-Ser33- $\beta$-catenin, active $\beta$-catenin. To determine the positively stained areas surrounding $\mathrm{Alp}^{+}$or bone surface areas, five fields of view per mouse per antibody were manually scored as either positive or negative staining. Dynamic bone histomorphometry was performed as previously (Chen et al., 2017). 8- $\mu \mathrm{m}$-thick longitudinal tibial sections were prepared. Unstained sections were used for assessment of mineral apposition rate (MAR, $\mu \mathrm{m} /$ day), whereas new bone formation was assessed by fluorescence microscopy of Calcein (green). Histomorphometric analysis was performed by a blinded observer using BioQuant OSTEO 2010 software (BioQuant Image Analysis Corporation, Nashville, TN).

\section{Statistics}

Numerical data was expressed as means \pm SD or SEM, and statistical analysis was performed by using the SPSS statistical package (IBM, North Castle, NY). We determined significance by Student's $t$ test or one-way ANOVA and Tukey-Kramer multiple comparisons test. Statistical significance was assessed at levels of $p<0.05$ and $p<0.01$. Experiments were independently repeated and the representative experiments are shown.

\section{Results}

RhoA/Rock activation by Wnt3a constrains $\beta$-catenin signaling

To determine the role of RhoA/Rock in Wnt/ $\beta$-catenin signaling, we used an established binding assay to examine the levels of GTP-bound (active) form in primary murine calvarial osteoblasts (PMCOBs). Stimulation with recombinant mouse Wnt3a resulted in timedependent and dose-dependent increases in the GTPRhoA levels in PMCOBs (Fig. 1a, b). Activation of RhoA by Wnt3a was confirmed in several cell lines, including C3H10T1/2 cells, a cell line of mouse embryonic fibroblasts, HEK293T cells, and L929 cells (Fig. S1A-C). The potential involvement of Lrp5/6 was assessed by treating the PMCOBs with recombinant mouse Dkk1 protein. A Wnt3a treatment increased the GTP-RhoA levels regardless of the presence or absence of Dkk1; however, Dkk1 inhibited both the basal and Wnt3a-induced increases in GTP-RhoA levels (Fig. 1c). Rac1 or Cdc42 antagonizes RhoA signaling in many cell types, and Rac1 activation by Wnt3a enhances $\beta$-catenin signaling (Wan et al. 2013; Wu et al. 2008). For these reasons, we also examined the potential involvement of Rac1/Cdc42 in the RhoA activation in response to Wnt3a. We found that Wnt3a activated the different small GTPases concurrently and independently in C3H10T1/2 cells (Fig. S1D-F). Thus, Wnt3a activates RhoA in both an Lrp5/6-dependent and Lrp5/6-independent manner, but in a Rac1- or Cdc42-independent manner.

We then explored the exact role of RhoA in Wnt/ $\beta$ catenin signaling by crossing mice that conditionally expressed the dominant negative form of RhoA (dnRhoA, T19N) or the constitutively active form of RhoA (caRhoA, G14V) with the mice expressing Cre recombinase under the control of the $2.3-\mathrm{kb}$ mouse collagen1 1 (Col1) promoter. This generated Col1-Cre; dnRhoA $A^{+/-}$or Col1-Cre;caRhoA ${ }^{+/-}$neonates and their control littermates. We then isolated PMCOBs from these mouse strains (P2 $\sim \mathrm{P} 5)$ and conducted western blot analyses to identify the cytosolic versus nuclear fractions of $\beta$-catenin. Activation of RhoA in the PMCOBs from the Col1-Cre;CaRhoA ${ }^{+/-}$neonates and its inactivation in PMCOBs from Col1-Cre; $d n R h o A^{+/-}$neonates were validated by determining the p-Rock2 levels (Fig. 1d, e). Activation (inactivation) of RhoA in PMCOBs slightly reduced (increased) the $\beta$-catenin in the wholecell lysate as well as in the cytosolic or nuclear fraction under basal conditions, whereas activation (inactivation) of RhoA notably suppressed (enhanced) the $\beta$-catenin accumulation in these cell fractions in response to Wnt3a (Fig. 1d, e). Likewise, the mRNA levels of Lef1, Cyclin D1, and Axin2, which are targets of Wnt/B-catenin signaling, were significantly decreased in PMCOBs of Coll-Cre;CaRhoA ${ }^{+/-}$neonates, or increased in PMCOBs of Col1-Cre; dnRhoA ${ }^{+-}$neonates, in either the presence or absence of Wnt3a (Fig. S2A, B). We also confirmed the role of RhoA in Wnt/ $\beta$-catenin signaling by overexpressing either caRhoA or RhoA siRNA (RhoA-si) in C3H10T1/2 cells (Fig. S2D, E). All these 
a

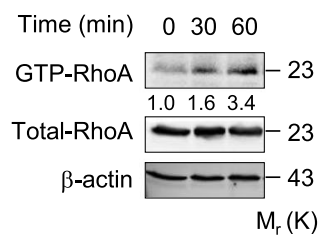

d

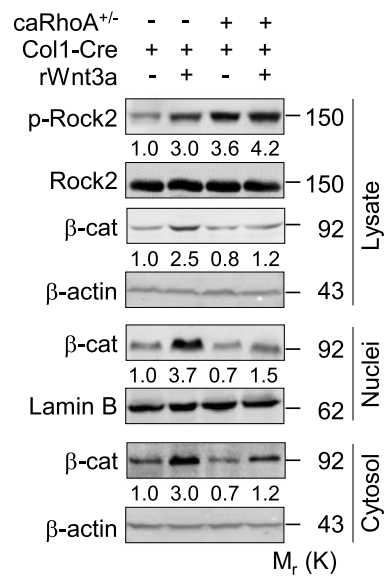

g

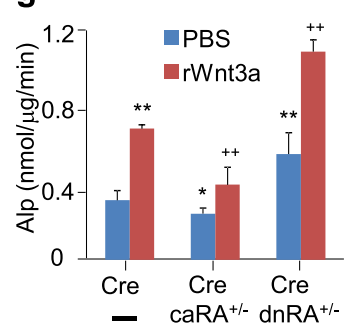

b

rWnt3a(ng/ml) 050100

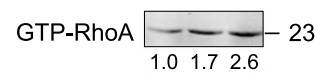

Total-RhoA $-1.01 .72 .6-23$

$\beta$-actin $-\longrightarrow-43$

$\mathrm{M}_{\mathrm{r}}(\mathrm{K})$

e

$\mathrm{dnRhoA}^{+-}-$- $^{+}+$

Col1-Cre ++++

rWnt3a - + - +

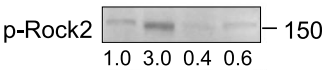

Rock2 1.03 .00 .40 .6

$\beta$-cat -92 -

$\begin{array}{llll}1.02 .42 .3 \quad 3.7 \\ \beta \text {-actin } & - & & \end{array}$

$\beta$-cat $=92$

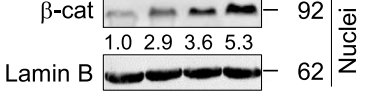

$\beta$-cat $\sim-92$

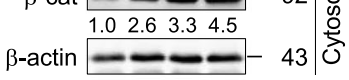

$M_{\mathrm{r}}(\mathrm{K})$

h

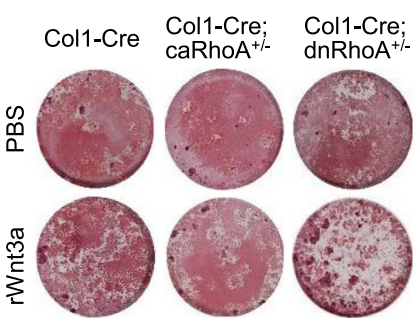

C

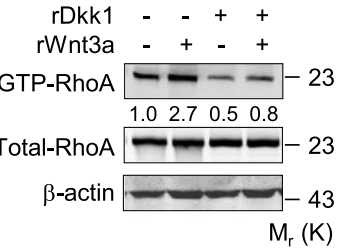

f

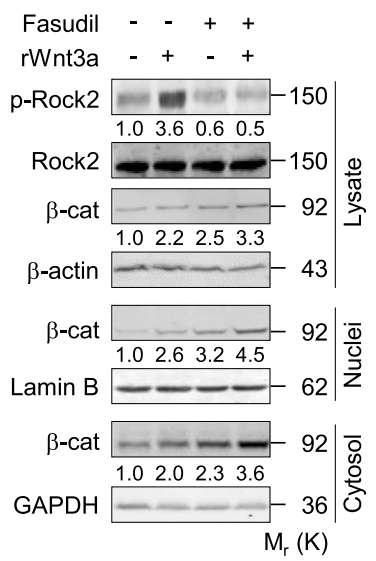

i

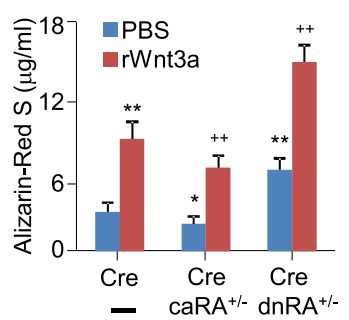

Fig. 1 RhoA/Rock constraints Wnt/B-catenin signaling and osteoblastic differentiation. a-c RhoA activation assays in primary murine calvarial osteoblasts (PMCOBs) stimulated with rWnt3a at $100 \mathrm{ng} / \mathrm{ml}$ or the indicated concentrations for the indicated times or 60 min in the presence or absence of recombinant Dkk1 (rDkk1) at $100 \mathrm{ng} / \mathrm{ml}$. d, e Western analyses of $\beta$-catenin in cytosolic and nuclear fractions of PMCOBs with the

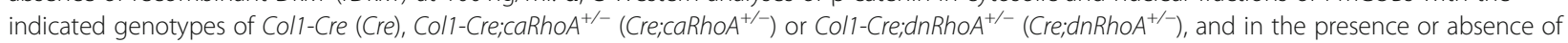
rWnt3a for $3 \mathrm{~h}$. $\mathbf{f}$ Western analyses of $\beta$-catenin ( $\beta$-cat) in cytosolic and nuclear fractions of PMCOBs treated with or without Fasudil at $20 \mu \mathrm{M}$ and stimulated with or without rWnt3a for $3 \mathrm{~h}$. $\mathbf{g}$-i Alp activity and mineralization nodule formation assays and their quantification in PMCOBs with the indicated genotypes and stimulated with or without $\mathrm{rWnt} 3 \mathrm{a}$ at $100 \mathrm{ng} / \mathrm{ml}$ for $48 \mathrm{~h}$ and $21 \mathrm{~d}$, respectively. Mean $\pm \mathrm{SEM}^{*}{ }^{*} p<0.05{ }^{* *+++} p<0.01$, $n=4$, Tukey-Kramer multiple comparisons test

findings are consistent with previous studies showing that inactivation of RhoA increases both the cytosolic and the nuclear levels of $\beta$-catenin (Peng et al. 2011; Shusterman et al. 2014; Miyagi et al. 2015). Thus, RhoA activation in response to Wnt3a constrains Wnt/ $\beta$-catenin signaling by decreasing both the cytosolic and nuclear levels of $\beta$-catenin.

Rock $1 / 2$ is the most extensively studied effectors of RhoA and is involved in many aspects of cell functions (Bishop and Hall 2000). We treated the wild type PMCOBs with a Rock2 inhibitor, Fasudil, to test whether Rock behaves essentially the same as $R h o A$ loss-of function in the regulation of $\mathrm{Wnt} / \beta$-catenin signaling. Fasudil treatment of PMCOBs slightly induced $\beta$-catenin expression in whole-cell lysates and in the cytosolic or nuclear fractions under basal conditions or robustly induced this expression in response to Wnt3a (Fig. 2f). Likewise, Fasudil treatment of PMCOBs consistently enhanced the mRNA levels of Lef1, Cyclin D1, and Axin2 (Fig. S2C). The specific role of Rock $1 / 2$ in Wnt/ $\beta$-catenin signaling was confirmed in $\mathrm{C} 3 \mathrm{H} 10 \mathrm{~T} 1 / 2$ cells using Rock1/2-si, a constitutively active form of Rock2 (caRock2), or another Rock1/2 inhibitor Y27632 (Fig. S3AC). Thus, Rock $1 / 2$ functions as a downstream effector of RhoA in the regulation of Wnt/ $\beta$-catenin signaling.

Wnt/ $\beta$-catenin signaling is the master regulator of BMMSC differentiation toward the osteoblastic lineage. We assessed the biological relevance of RhoA/Rock in 


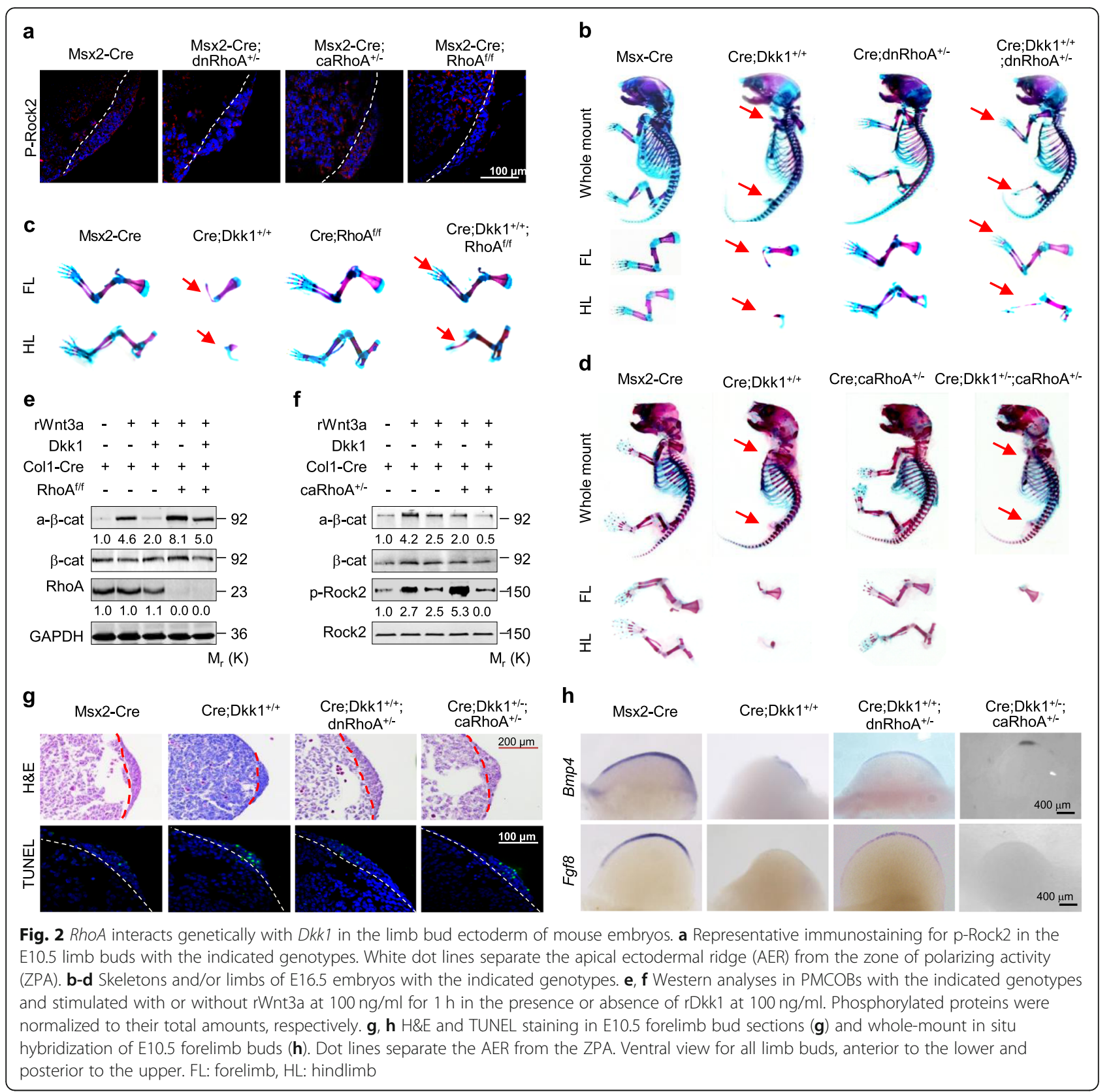

the regulation of $\mathrm{Wnt} / \beta$-catenin signaling by testing PMCOBs from Col1-Cre, Col1-Cre;caRhoA ${ }^{+/}$, and Col1$\mathrm{Cre} ; d n R h o A^{+/-}$neonates for their ability to undergo osteoblastic differentiation in response to Wnt3a. Activation of RhoA in PMCOBs significantly reduced the activity of alkaline phosphatase (Alp), an osteoblast marker, and the formation of mineralized nodules in either the presence or absence of Wnt3a, while inactivation enhanced these responses (Fig. 1g-i). These findings are consistent with previous studies showing that Rock negatively regulates osteoblastic differentiation and bone formation (Ohnaka et al. 2001; Kanazawa et al. 2009,
2010). Thus, RhoA/Rock negates Wnt/ $\beta$-catenin signaling, thereby suppressing osteoblastic differentiation.

\section{RhoA interacts genetically with Dkk1 in the AER}

We established the genetic role of RhoA in the regulation of Wnt/ $\beta$-catenin signaling using RhoA loss-offunction or gain-of-function at the apical ectodermal ridge (AER) of the mouse embryonic limb bud, where Wnt signaling is critical for limb outgrowth via $\beta$ catenin (Barrow et al. 2003). Specifically, we generated Msx2-Cre, Msx2-Cre;dnRhoA ${ }^{+/-}$, Msx2-Cre;caRhoA ${ }^{+-}$, and $M s \times 2-C r e ; R h o A^{f / f}$ mouse embryos, taking advantage 
of the fact that Msx2-Cre is expressed in the limb bud ectoderm that gives rise to the AER (Sun et al. 2000). We used immunostaining for p-Rock2 to validate the genetic modifications in the E10.5 limb buds and found a significantly abolished or diminished p-Rock2 expression in the AER of the Msx2-Cre; $d n R h o A^{+-}$or $M s \times 2-C r e ; R h o A^{f / f}$ embryos, respectively, but a robust enhancement of expression in the AER of the Msx2-Cre; caRhoA ${ }^{+/-}$embryos (Fig. 2a).

The Msx2-Cre;dnRhoA ${ }^{+/}$, Msx2-Cre;caRhoA ${ }^{+/-}$, and Msx2-Cre; RhoA $A^{f / f}$ embryos at E16.5 displayed similarly long and normally structured forelimbs and hindlimbs, with autopods, zeugopods, and stylopods, when compared with their control littermates (Fig. 2b-d). We therefore generated $M s x 2-C r e ; d n R h o A^{+/-} ; D k k 1^{+/+} \mathrm{em}-$ bryos by crossing the female $d n R h o A^{+/-} ; D k k 1^{+/-}$mice with male Msx2-Cre; $k_{k} 1^{+/-}$mice. The Msx2-Cre; $D k k 1^{+/+}$embryos at E16.5 lacked all hindlimb structures, including the autopods, zeugopods, and stylopods, and displayed truncations at various levels in the forelimbs (Fig. 2b-d), consistent with our previous findings (Wu et al. 2008). Remarkably, the Msx2-Cre; $d n R h o A^{+/-}$; $D k k 1^{+/+}$embryos (6/6) exhibited normal forelimbs and varying lengths of hindlimbs but entirely lacked autopods (Fig. 2b). To confirm the specificity of $d n R h o A$, we then crossed female $R h o A^{f f f} ; D k k 1^{+/-}$mice with male Msx2-Cre;Rho $A^{f /+} ; \mathrm{Dkk1}^{+/-}$mice to generate Msx2-Cre; $R h o A^{f f f} ; D k k 1^{+/+}$embryos that had a conditional knockout of $R h o A$ and overexpression of $D k k 1$ in the limb bud ectoderms. The Msx2-Cre;RhoA $A^{f / f} ; D k k 1^{+/+}$embryos (4/4) exhibited the same limb phenotypes as the Msx2-Cre; $d n R h o A^{+/-} ; D k k 1^{+/+}$embryos, including the varying hindlimb length and lack of autopods (Fig. 2c). Thus, RhoA loss-of-function was sufficient to rescue the limb defects caused by $D k k 1$ overexpression in the limb bud ectoderm.

We explored a specific role for RhoA in Wnt/ $\beta$-catenin signaling by determining whether the two molecules genetically interact in the AER. To do this, we crossed $\mathrm{caRhoA}^{+/-}$and Ms $\times 2-\mathrm{Cre} ; \mathrm{Dkk1^{+/- }}$ mice to generate embryos that expressed both caRhoA and Dkk1 in the AER. The resulting embryos with Msx2-Cre and one copy of either the Dkk1 or caRhoA allele (Msx2-Cre; $D k k 1^{+/-}$or $\left.M s x 2-C r e ; C a R h o A^{+/-}\right)$did not show any obvious limb phenotype changes; however, the doubleheterozygous embryos (Msx2-Cre; $D k k 1^{+/-} ; \mathrm{caRhoA}^{+/-}$) (6/6) all failed to develop hindlimbs and nearly all the forelimbs (10/12 from 6 embryos) lacked structures distal to the scapula; these phenotypes are identical to those of the Msx2-Cre;Dkk1 $1^{+/+}$or Msx2-Cre; $\beta$-catenin ${ }^{n / f} \mathrm{em}-$ bryos (Fig. 2d). One embryo was an exception and lacked forelimb structures distal to the humerus. Overall, $R h o A$ was confirmed to interact genetically with $D k k 1$ in regulating $\mathrm{Wnt} / \beta$-catenin signaling and limb outgrowth.
RhoA regulation of the limb outgrowth depends on Wnt/ $\beta$-catenin signaling

We examined whether the effect of RhoA on limb bud outgrowth depends on $\beta$-catenin by crossing female Msx2Cre; $\beta$-catenin ${ }^{f /+}$ with male $d n R h o A^{+/} ; \beta$-catenin ${ }^{\text {fff }}$ mice to generate $M s x 2 C r e ; \beta$-catenin ${ }^{n / f} ; d n R h o A^{+/-}$embryos, taking advantage of the maternal germline transmission of Msx2-Cre. Unlike the Msx2-Cre; $d n R h o A^{+/-}$; $D k k 1^{+/+}$embryos, the Msx2-Cre;dnRhoA ${ }^{+/} ; \beta$-catenin ${ }^{\text {n/f }}$ embryos (6/6) at E16.5 still lacked all hindlimb structures and exhibited truncations at various levels in the forelimbs; these defects were identical to those in the Msx2-Cre; $\beta$-catenin ${ }^{n / f}$ embryos (Fig. S4A). We also generated the $M s x 2-C r e ; D k k 1^{+/+} ; \beta$-catenin ${ }^{n / f}$ and $M s x 2-$ Cre;dnRhoA ${ }^{+/-} ; D k k 1^{+/+} ; \beta$-catenin ${ }^{n / f}$ embryos at E16.5 to determine whether $d n R h o A$ would restore the $M s x 2-C r e$; $D k k 1^{+/+}$phenotype in the absence of $\beta$-catenin. As expected, the Msx2-Cre;Dkk1 ${ }^{+/+} ; \beta$-catenin ${ }^{n / f}$ embryos lacked both forelimbs and hindlimbs and displayed the more severe limb phenotypes than either the Msx2-Cre; $D k k 1^{+/+}$or $M s x 2-C r e ; \beta$-catenin ${ }^{n / f}$ embryos. However, However, expression of $d n R h o A$ in AER did not result in any rescue of the limb phenotypes of $\mathrm{Ms} \times 2-\mathrm{Cre} ; \mathrm{Dkk1^{++ }}$; $\beta$ - atenin $^{n / f}$ embryos (Fig. S4B). Thus, RhoA genetically regulates limb outgrowth, depending on $\beta$-catenin signaling.

We then determined the molecular events of RhoA loss-of-function or gain-of function in regulating the limb phenotypes caused by $D k k$-overexpression in AER by performing western blot assays in PMCOBs isolated from Col-Cre, Col-Cre;RhoA $A^{f / f}$ or Col-Cre;CaRhoA ${ }^{+/-}$neonates. Wnt3a significantly increased the levels of active $\beta$-catenin, and Dkk1 robustly diminished this induction by Wnt3a in Col-Cre PMCOBs. Importantly, deletion of RhoA robustly increased the levels of active $\beta$-catenin and activation of RhoA robustly decreased those levels, whereas deletion of RhoA significantly restored and activation of RhoA significantly potentiated the Dkk1negated active $\beta$-catenin in the Col-Cre; $\mathrm{RhoA}^{\mathrm{f} / \mathrm{f}}$ and $\mathrm{Col}$ Cre;caRhoA ${ }^{+/-}$PMCOBs in response to Wnt3a (Fig. 2e, f). Thus, RhoA loss-of-function was sufficient to restore, and gain-of-function was sufficient to potentiate, the Dkk1-reduced $\beta$-catenin levels.

We sought to gain further insights into the role of RhoA in the limb bud ectoderm by performing histological and TUNEL assays and in situ hybridization in E10.5 embryos. Consistent with the limb truncation phenotype, the $M s \times 2-C r e ; D k k 1^{+/+}$embryos displayed noticeably smaller forelimbs and hindlimb buds than their control littermates; however, overexpression of $d n R h o A$ in the AER of $M s x 2-C r e ; D k k 1^{+/+}$embryos significantly increased the sizes of both fore- and hindlimb buds, while overexpression of $c a R h o A$ in the AER of Msx2$C r e ; D k k 1^{+-}$embryos significantly decreased these bud 
sizes (Fig. 2g and Fig. S5A). Likewise, Likewise, cell apoptosis in the AER was significantly lower in $M s \times 2$ Cre; $D k k 1^{+/+} ; d n R h o A^{+/-}$embryos than in Msx2-Cre; $D k k 1^{+/+}$embryos, whereas cell apoptosis was similar in

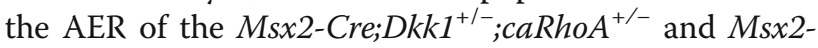
Cre; $D k k 1^{+/+}$embryos (Fig. $2 g$ and Fig. S5A, C).

The striking morphological alterations caused by overexpression of $d n R h o A$ in $M s x 2-C r e ; D k k 1^{+/+}$embryos or of $c a R h o A$ in $M s x 2-C r e ; D k k 1^{+/-}$embryos prompted us to examine the potential regulation of RhoA at the molecular level. We performed in situ hybridization for fibroblast growth factor 8 (Fgf8) in the AER and bone morphogenetic protein $4(B m p 4)$ in the distal ventral ectoderm; both of these are target genes of $\mathrm{Wnt} / \beta$-catenin signaling. As expected, the Msx2-Cre;Dkk1 ${ }^{+/+} \mathrm{em}-$ bryos expressed only residual Bmp4 and no Fgf8 in the forelimb of E10.5 (34-35 somites) embryos (Fig. 2h and Fig. S5B, D). By contrast, overexpression of $d n R h o A$ largely restored the expression of $F g f 8$ and Bmp4 in the Msx2-Cre; $D k k 1^{+/+}$background, whereas overexpression of caRhoA robustly diminished the expression of both Fgf8 and Bmp4 in the Msx2-Cre;Dkk1 ${ }^{+-}$background (Fig. 2h and Fig. S5B, D). Both morphologically and molecularly, RhoA interacts with Wnt/ $\beta$-catenin signaling to regulate the limb truncation phenotype caused by $D k k 1$ overexpression in the AER.

\section{RhoA/Rock activates Gsk3 $\beta$ to destabilize $\beta$-catenin}

We next examined the downstream events of RhoA/ Rock in the regulation of Wnt/ $\beta$-catenin signaling. Both protein kinase B (Akt) and Gsk3 $\beta$ have been suggested to function as effectors of RhoA/Rock signaling and both are involved in the phosphorylation of $\beta$-catenin (Papakonstanti et al. 2007; Kim et al. 2017). Therefore, we performed western blot analyses with antibodies specific for $\mathrm{p}$-Ser552- $\beta$-catenin (an Akt phosphorylation site) and p-Ser473-Akt (associated with stimulation of Akt activity). We found that neither RhoA-si nor caRhoA significantly affected these phosphorylation events in C3H10T1/2 cells (Fig. S6A, B). Thus, Akt- $\beta$-catenin signaling axis is not the target of RhoA/Rock.

We next examined the relevance of Gsk3 $\beta$ with antibodies specific for p-Ser33- $\beta$-catenin (a Gsk3 $\beta$ phosphorylation site) and p-Ser9-Gsk3 $\beta$ and p-Tyr216-Gsk3 $\beta$ (associated with inhibition and enhancement of Gsk3 $\beta$ activity, respectively). Wnt3a increased $\mathrm{p}-\mathrm{Ser} 9-\mathrm{Gsk} 3 \beta$ or decreased $\mathrm{p}$-Ser33- $\beta$-catenin in a time-dependent manner within $60 \mathrm{~min}$ of stimulation, but time-dependently induced an increase in p-Tyr216-Gsk3 $\beta$ (Fig. 3a), which is essential for full kinase activity of Gsk3 $\beta$ (Wu and Pan 2010; ter Haar et al. 2001). Importantly, RhoA or the combination of Rock1 and Rock2 siRNA suppressed both p-Ser33- $\beta$-catenin and p-Tyr216-Gsk3 $\beta$ in the basal conditions and in response to Wnt3a, without affecting
p-Ser9-Gsk3 $\beta$ (Fig. 3b, c and Fig. S6C, D). By contrast, either caRhoA or caRock2 robustly enhanced both the p-Tyr216-Gsk3 $\beta$ and p-Ser33- $\beta$-catenin levels (Fig. S6E). Thus, RhoA/Rock destabilizes $\beta$-catenin while concomitantly activating Gsk $3 \beta$.

We then explored the potential importance of Gsk3 $\beta$ phosphorylation at Tyr216 in the destabilization of $\beta$ catenin by expressing a Gsk3 $\beta$ variant that has the tyrosine residue mutated to phenylalanine (Y216F, phospho-null mutation) and evaluating the capacity to mediate $\mathrm{Wnt} / \beta$-catenin signaling. In the absence of Wnt3a, the wildtype Gsk3 $\beta$ (WT) decreased the $\beta$ catenin levels by approximately $50 \%$ and Lef1-luciferase expression by approximately $60 \%$ over the vector, whereas the Y216F variant of Gsk3 $\beta$ caused $100 \%$ and $85 \%$ increases, respectively, over the WT levels. In the presence of Wnt3a, the WT completely abolished this induction of $\beta$-catenin levels and Lef1-luciferase expression, but the Y216F variant only minimally affected the induction of $\beta$-catenin levels and Lef1-luciferase expression (Fig. 3d, e). Thus, Gsk3 $\beta$ phosphorylation at Tyr216 is responsible for the RhoA/Rock-mediated Wnt/B-catenin signaling.

\section{Jak1/2 mediates RhoA/Rock-induced Gsk3 $\beta$ activation and $\beta$-catenin destabilization}

Rock $1 / 2$ is a serine/threonine kinase; however, it is unlikely to be directly responsible for phosphorylation of Gsk3 $\beta$ at Tyr216. We explored the underlying mechanism by which RhoA/Rock induces Gsk $3 \beta$ phosphorylation at Tyr216 by investigating the potential relevance of the Jak tyrosine kinase that physically interacts with and is activated by Rock (Huang et al. 2013; Orgaz et al. 2014). In contrast to the relatively ubiquitous expression of Jak1 and Jak2, Jak3 expression is restricted to hematopoietic cells, vascular smooth muscle cells, and endothelium (Müller et al. 2005). We therefore examined the role of Jak $1 / 2$ in the following experiments. Western blot analyses were performed using antibodies specific for p-Tyr1022-Jak1 and p-Tyr1007-Jak2, the prerequisites for Jak1 and Jak 2 activation, respectively. Concomitant with the induction of Gsk3 $\beta$ phosphorylation at Tyr216, Wnt3a increased both p-Jak1 and p-Jak2 over the control within $120 \mathrm{~min}$ of stimulation (Fig. 3f). Wnt3a robustly increased p-Jak1/2 levels in either the presence or absence of Dkk1, although Dkk1 decreased both the basal and Wnt3a-induced p-Jak1/2 levels (Fig. S7A). Likewise, the activation of Jak1 and Jak2 was essentially abolished by the expression of either a RhoA siRNA or a combination of Rock1 and Rock2 siRNA (Fig. 3g and Fig. S7B). By contrast, this activation was robustly enhanced by the expression of either caRhoA or caRock2 (Fig. 3h). 


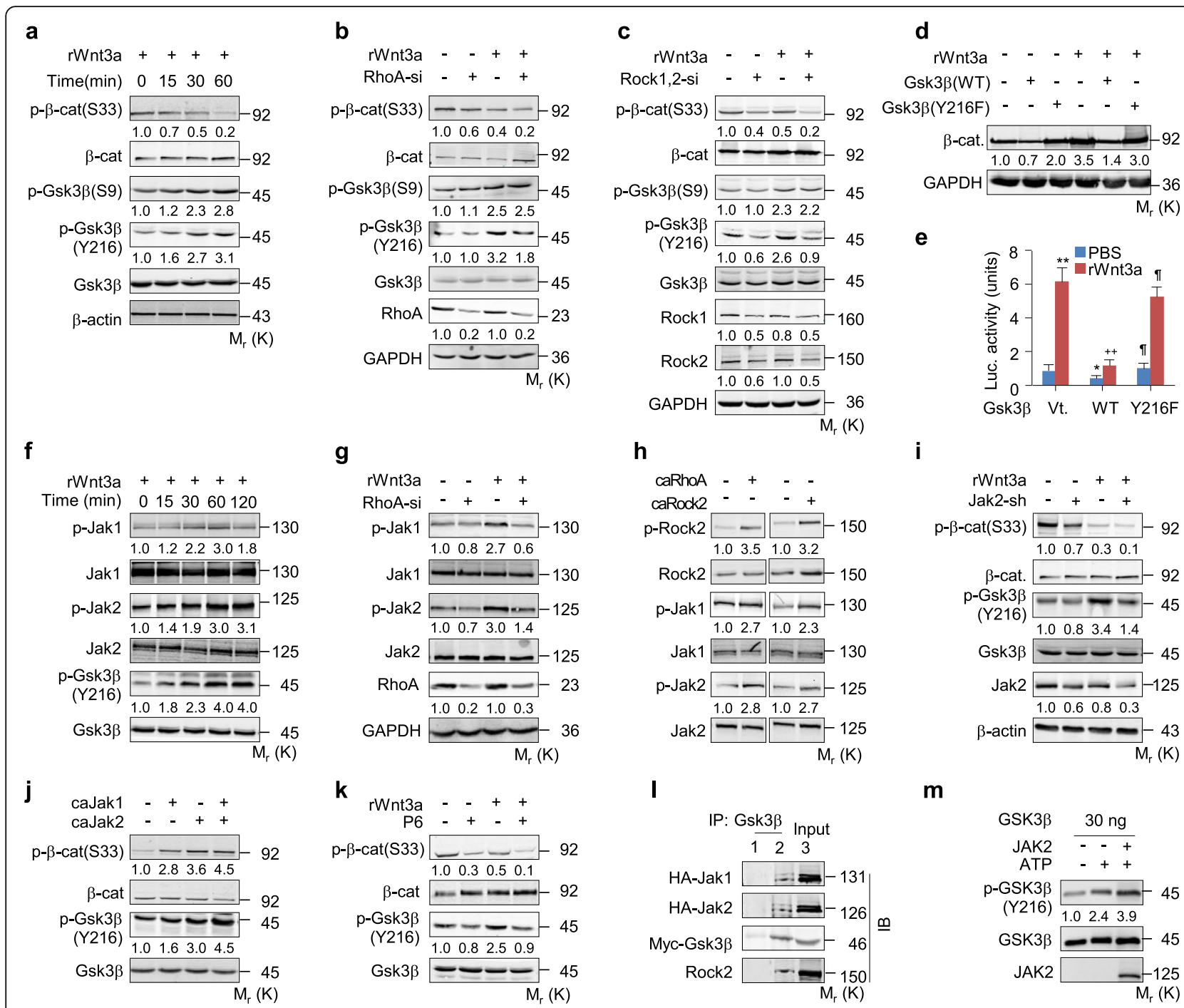

Fig. 3 RhoA/Rock activates Jak1/2 and Gsk3ß to destabilize $\beta$-catenin. a-c Western analyses in C3H10T1/2 cells transfected with or without RhoAsi or Rock1 + Rock2 siRNA (Rock1,2-si) and treated with or without rWnt3a at $100 \mathrm{ng} / \mathrm{ml}$ for the indicated time or $1 \mathrm{~h}$. d, e Western or Lef1luciferase expression analyses in C3H10T1/2 cells transfected with Gsk3 3 variants and treated with rWnt3a for 6 or 48 h, respectively. $\mathbf{f}-\mathbf{k}$ Western analyses in C3H10T1/2 cells transfected with RhoA-si, caRhoA, caRock2, caJak1/2, infected with lentiviral Jak2-shRNA (Jak2-sh), or treated with P6 at $50 \mathrm{nM}$, followed by incubation with rWnt3a for the indicated times or $1 \mathrm{~h}$. I Co-immunoprecipitation by using IgG1 or Gsk3 3 antibody in 293 cells transfected with HA-Jak1/2 and Myc-Gsk33. $\mathbf{m}$ In vitro phosphorylation of GSK3ß protein by active JAK2 in kinase assay buffer with or without ATP. Phosphorylated proteins were normalized to their total amounts, respectively. Mean $\pm \mathrm{SD},{ }^{*},{ }^{\uparrow} p<0.05,{ }^{* *},++p<0.01, \mathrm{n}=4$, TukeyKramer multiple comparisons test

We also addressed the role of Jak1/2 in Wnt/ $\beta$-catenin signaling by knockdown of Jak1/2 with specific shRNAs. Jak1-shRNA and Jak2-shRNA reduced both the pTyr216-Gsk3 $\beta$ and the p-Ser33- $\beta$-catenin levels under the basal conditions and in response to Wnt3a (Fig. 3i and Fig. S7C). By contrast, the constitutively active form of Jak1 (caJak1) or Jak2 (caJak2) apparently increased both the p-Tyr216-Gsk3 $\beta$ and p-Ser33- $\beta$-catenin levels (Fig. 3j). In addition, both Jak1- and Jak2-shRNAs increased the Lef1-luciferase expression with or without Wnt3a stimulation (Fig. S7D, E), whereas Pyridone 6 (P6), a pan-Jak inhibitor, at $50 \mathrm{nM}$, increased the Lef1- luciferase expression and decreased the p-Tyr216-Gsk3 $\beta$ and $\mathrm{p}$-Ser33- $\beta$-catenin with or without Wnt3a (Fig. 3k and Fig. S7F).

We explored the potential physical interactions between Rock, Jak, and Gsk3 $\beta$ by co-immunoprecipitation experiments using cell lysates from C3H10T1/2 cells that co-expressed HA-tagged Jak1/2 and Myc-tagged Gsk3 $\beta$. The protein complex precipitated with a Gsk3 $\beta$ antibody contained the exogenous HA-tagged Jak $1 / 2$ as well as the endogenous Rock2, in addition to Myctagged Gsk3 $\beta$, as expected (Fig. 3l). The physical interaction between Jak and Gsk3 $\beta$ was further demonstrated 
by immunostaining in $\mathrm{C} 3 \mathrm{H} 10 \mathrm{~T} 1 / 2$ cells that transiently expressed HA-Jak2 and Flag-tagged Gsk3 $\beta$ (Fig. S7G). We also investigated whether Gsk3 $\beta$ was phosphorylated at Tyr216 by Jak by in vitro phosphorylation assays using purified active human JAK2 and GSK3 $\beta$. In the absence of JAK2, recombinant human GSK3 $\beta$ underwent more autophosphorylation at Tyr216 upon incubation with ATP. Importantly, when GSK3 $\beta$ was incubated with JAK2 in the presence of ATP, significantly more GSK3 $\beta$ phosphorylation occurred at Tyr216 (Fig. 3m). Taken together, the data indicate that RhoA/Rock activates Jak1/ 2 to directly phosphorylate Gsk3 $\beta$ at Tyr216, resulting in Gsk $3 \beta$ activation and $\beta$-catenin destabilization.

\section{Jak1/2-mediated GSK3 $\beta$ activation participates in osteoblastogenesis}

We confirmed a role for the Rock/Jak/Gsk3 $\beta$ signaling axis in regulating $\mathrm{Wnt} / \beta$-catenin signaling by immunostaining for Alp (osteoblast marker), p-Rock2, p-Jak2, pTyr216-Gsk3 $\beta$, and active $\beta$-catenin in longitudinal tibia sections of Col1-Cre, Col1-Cre;dnRhoA ${ }^{+/}$, and Col1-Cre; $\mathrm{caRhoA}^{+/-}$mice. Inactivation or activation of RhoA in osteoblasts was validated by detecting the numbers of $\mathrm{p}$ Rock $2^{+}$cells in the $\mathrm{Alp}^{+}$osteoblast populations (Fig. S8A, B). The numbers of $\mathrm{p}-J \mathrm{ak} 2^{+}$and $\mathrm{p}$-Tyr216-Gsk $3 \beta^{+}$ osteoblasts showed a robust decrease in tibias from Col1-Cre; $d n R h o A^{+/-}$mice and a robust increase in tibias from Col1-Cre;caRhoA ${ }^{+/-}$mice when compared to the Col1-Cre mouse tibias. By contrast, the numbers of active $\beta$-catenin ${ }^{+}$osteoblasts were significantly increased in the Col1-Cre; $d n R h o A^{+/-}$tibias and significantly decreased in the Col1-Cre;caRhoA ${ }^{+/-}$tibias (Fig. 4a-d).

We investigated the physiological relevance of $\mathrm{p}$-Jak and p-Tyr216-Gsk3 $\beta$ activation by Wnt3a using osteoblastic differentiation assays. C3H10T1/2 cells were transfected with either wild type or Gsk3 $\beta$-Y216F or treated with $50 \mathrm{nM}$ P6 and then examined for their ability to undergo osteoblastic differentiation in response to Wnt3a. Wildtype Gsk3 $\beta$ transfection reduced the Wnt3a-induced Alp activity and mineralized nodule formation by $63 \%$ and $89 \%$, respectively, while transfection with the Y216F mutant Gsk3 $\beta$ decreased the Wnt3ainduced Alp activity and mineralized nodule formation by only $23 \%$ and $20 \%$, respectively (Fig. $4 \mathrm{e}-\mathrm{g}$ ). Consistently, the inhibition of Jak by P6 robustly increased the Alp activity and mineralized nodule formation with or without the addition of Wnt3a (Fig. 4h-j). Thus, RhoA/ Rock destabilizes $\beta$-catenin by activating Jak and Gsk3 $\beta$ during the regulation of osteoblastogenesis.

RhoA loss-of-function or gain-of-function affects the bone mass in the adult mice

Overexpression of $d n R h o A$ or caRhoA caused severe limb phenotypes in the genetic background of $M s x 2-C r e$;
$D k k 1^{+/+}$or $M s \times 2-C r e ; D k k 1^{+/-}$, respectively. However, overexpression of $d n R h o A$ or caRhoA alone resulted in no apparent limb phenotype changes in the otherwise wild type background. This observation led us to speculate that inactivation or activation of RhoA in the wild type background underscores both the robustness of the $\mathrm{Wnt} / \beta$-catenin signaling system and the sensitivity of the AER to a threshold level of $W n t / \beta$-catenin signaling.

We determined whether RhoA/Rock-mediated Wnt/ $\beta$ catenin signaling is sufficiently robust or sensitive for regulation of bone homeostasis by generating 8-weekold male or female Col1-Cre; dnRhoA ${ }^{+-}$or Col1-Cre; $\mathrm{caRhoA}^{+/-}$mice and their control littermates. Microcomputed tomography $(\mu \mathrm{CT})$ analyses and threedimensional reconstruction of the proximal tibias demonstrated that inactivation of RhoA in pre-osteoblasts increased the bone volume/trabecular volume (BV/TV), trabecular thickness (Tb.Th), and trabecular number (Tb.N) by $75 \%, 33 \%$, and $56 \%$, respectively, and decreased the trabecular separation (Tb.Sp) by $35 \%$. By contrast, activation of RhoA in pre-osteoblasts decreased the BV/TV, Tb. Th, and Tb.N by $47 \%, 22 \%$, and $35 \%$, respectively, and increased the Tb.Sp by $65 \%$ (Fig. 5a, b). Hematoxylin-eosin (H\&E) staining of longitudinal sections of the proximal tibias consistently revealed that inactivation of RhoA in pre-osteoblasts caused an apparent increase, while activation caused a decrease, in the bone volume, osteoblast number/bone surface (OB.N/BS), and osteoblast surface/bone surface (Ob.S/ BS) (Fig. 5c, d). By contrast, tartrate-resistant acid phosphatase (Trap)-stained sections indicated that neither inactivation nor activation of RhoA in pre-osteoblasts significantly affected the parameters of osteoclast number/bone surface (Oc.N/BS) and osteoclast surface/bone surface (Oc.S/BS) in Col1-Cre;dnRhoA ${ }^{+/-}$or Col1-Cre; $\mathrm{caRhoA}^{+/-}$mice, respectively (Fig. 5e, f). Thus, RhoA is essential for maintaining the bone homeostasis.

Consistent with the observed alterations in bone mass, immunohistochemical staining of the proximal tibia sections indicated that inactivation of RhoA in preosteoblasts significantly increased, while activation decreased, the numbers of $\mathrm{Alp}^{+}$osteoblasts surrounding the trabecular bone (Fig. S9A, B, D). By contrast, qPCR analyses of mRNA levels of osteoblast markers, including $A l p$, bone sialoprotein (Bsp), and Runx2, in femurs revealed that inactivation of RhoA robustly increased, while activation robustly decreased, these mRNA levels (Fig. 5g). Analyses of Lef1, Cyclin D1, and Axin2 mRNA levels in femurs persistently indicated that inactivation of RhoA robustly increased, and activation robustly decreased, the expression of these Wnt $/ \beta$-catenin signaling targets (Fig. 5h), consistent with the immunostaining of Lef1 in proximal tibia sections (Fig. S9A, C, E). Furthermore, dynamic bone histomorphometry with calcein 


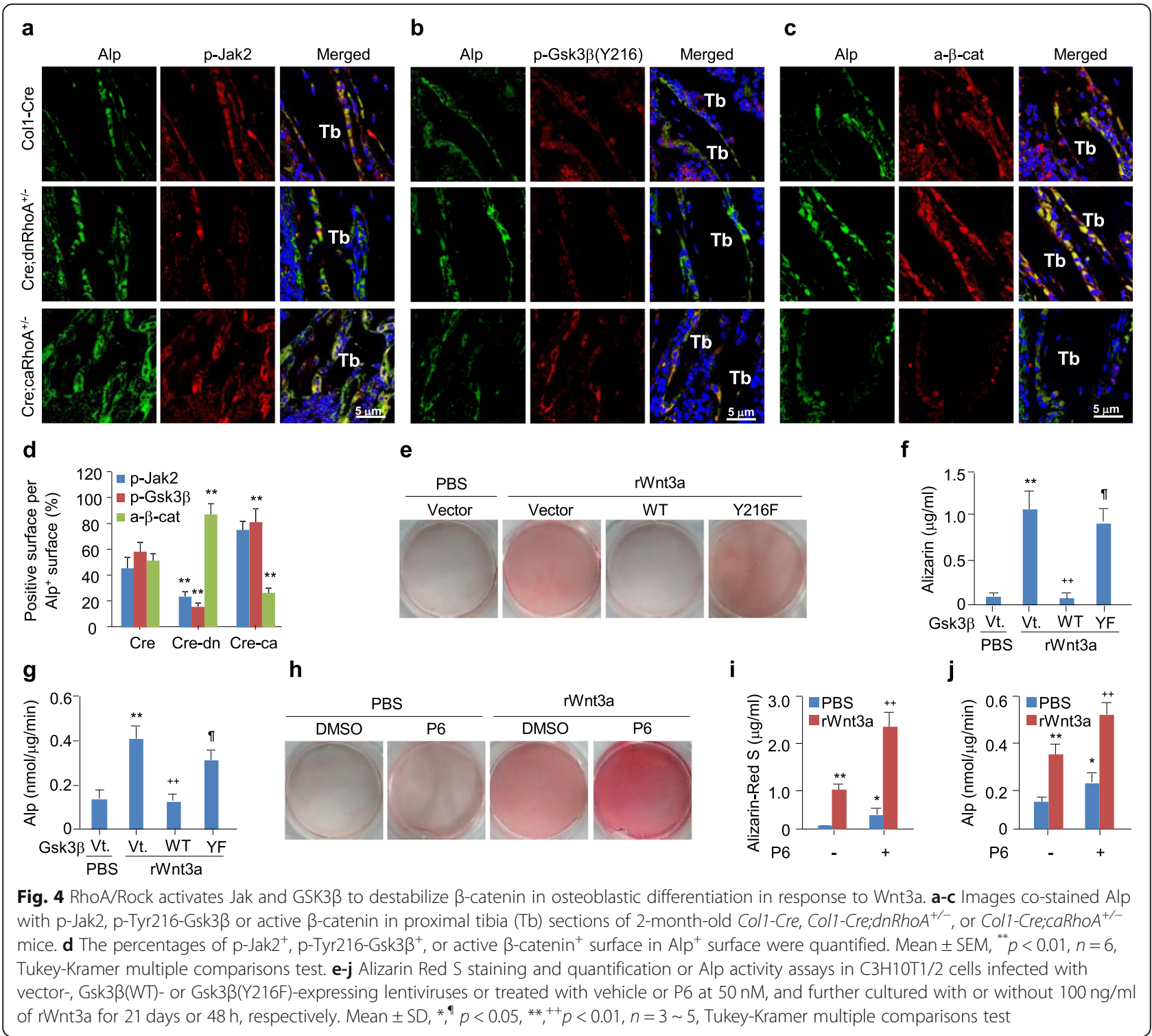

labeling indicated that inactivation of RhoA increased the bone formation rate/bone surface (BFR/BS), mineral apposition rate (MAR), and double-labeled surface/bone surface (dLS/BS) by $82 \%, 55 \%$, and $61 \%$, respectively, whereas activation of RhoA decreased BFR/BS, MAR, and dLS/BS by $47 \%, 38 \%$, and $31 \%$, respectively (Fig. 5i, j).

Serological analyses of bone metabolism markers indicated that inactivation of RhoA in pre-osteoblasts caused a significant increase, while activation caused a significant decrease, in the levels of bone formation markers, including procollagen type $1 \mathrm{~N}$-terminal propeptide (P1NP) and osteocalcin (OCN), whereas no effect was observed on the levels of bone resorption markers, including carboxyterminal collagen cross-links (CTX) and TRAP (Fig. S9F-I). Taken together, these data showed that inactivation or activation of RhoA in pre-osteoblasts affects bone formation but not bone resorption, possibly by regulating $\mathrm{Wnt} / \beta$-catenin signaling.

RhoA/Rock activity is inversely correlated with $\beta$-catenin signaling activity in BMMSCs from elderly human subjects Low activity of $\mathrm{Wnt} / \beta$-catenin signaling in BMMSCs contributes to the pathogenesis of type II osteoporosis or age-dependent bone loss (Jing et al. 2018; Manolagas and Almeida 2007). We used the bone marrow smears from young $(n=3,28-, 32-, 33-$ year-old) or elderly $(n=$ 4, 74-, 78-, 82-, and 84-year-old) male patients and the tibial sections from 5- or 10-month-old male mice, with normal bone volumes or aging-associated bone loss, respectively, to determine the potential relevance between RhoA/Rock and Wnt/ $\beta$-catenin signaling. We then 


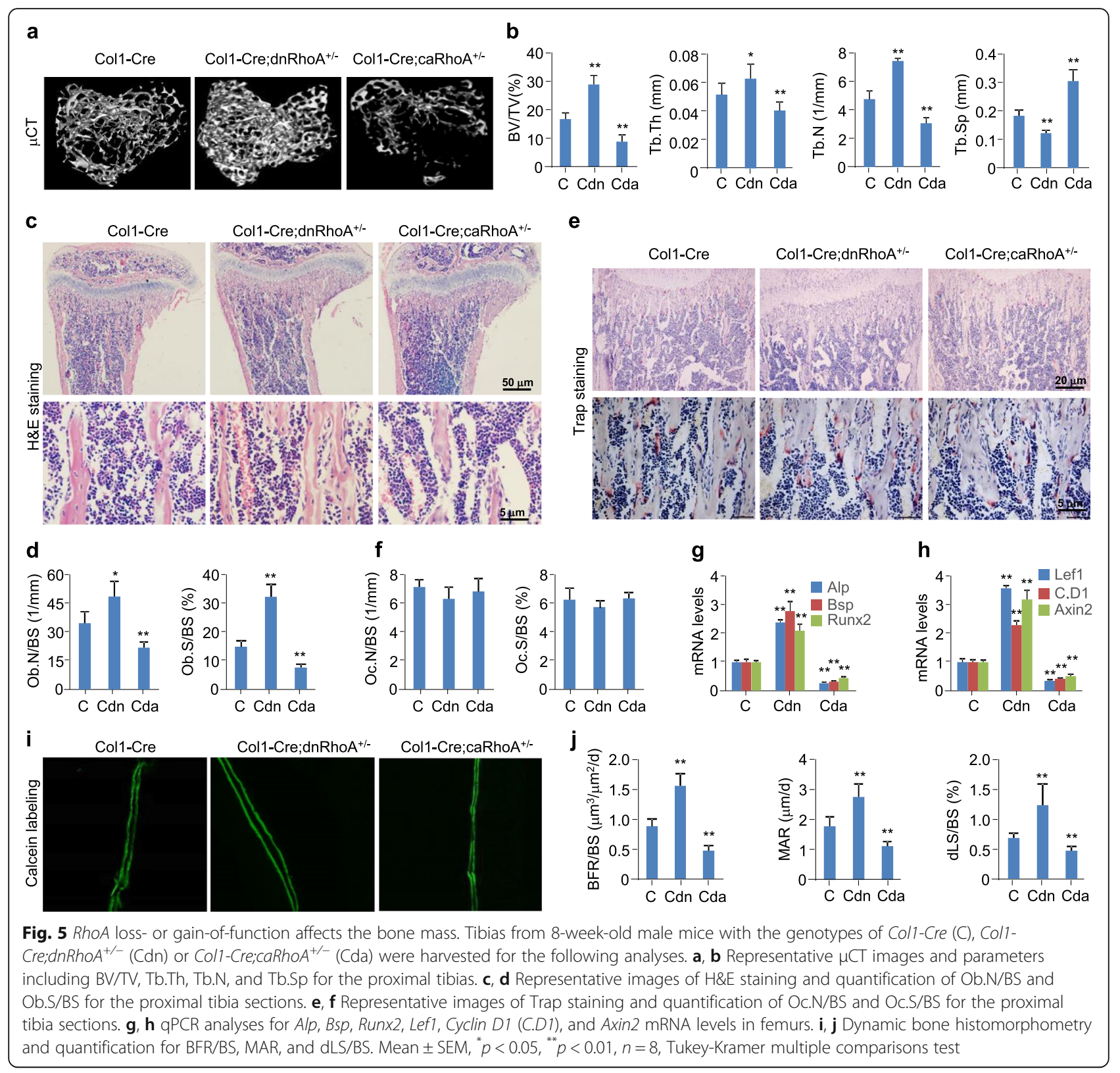

performed the immunostaining of $\mathrm{p}$-Rock2, $\mathrm{p}$-Jak1, $\mathrm{p}$ Tyr216-Gsk3 $\beta$, p-Ser33- $\beta$-catenin ( $\mathrm{p}-\beta$-cat, an inactive form of $\beta$-catenin), and non-p-Ser45- $\beta$-catenin (a- $\beta$-cat, an active form of $\beta$-catenin), and quantified the mRNA levels of Lef1, Cyclin D1, and Axin2, the targets of Wnt/ $\beta$-catenin signaling, and of Runx2, a osteoblastic gene marker.

NESTIN-positive $\left(\mathrm{NESTIN}^{+}\right)$BMMSCs were scattered

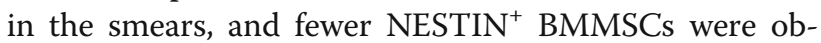
tained from the elderly men than from the young men. The immunosignals derived from p-ROCK2-, p-JAK1, pTyr216-GSK3 $\beta$, $p$ - $\beta$-CATENIN-, and active $\beta$-CATENIN were robustly detected, and the apparently overlapping signals between NESTIN and other molecules were readily observed in the cytoplasm of the BMMSCs (Fig. 6a-e). The NESTIN ${ }^{+}$BMMSCs from the elderly men displayed considerably more p-ROCK $2^{+}$, p-JAK $1^{+}$, p-Tyr216-GSK3 $\beta^{+}$and $\mathrm{p}-\beta$-CATENIN ${ }^{+}$cells but fewer active $\beta$-CATENIN ${ }^{+}$cells than were detected in the BMMSCs from the young men (Fig. 6a-f). Consistently, considerably lower LEF1, CYCLIN D1, AXIN2 and RUNX2 mRNA levels were detected in the BMMSCs from the elderly men than from the young men (Fig. $6 \mathrm{~g})$. Likewise, Nestin ${ }^{+}$BMMSCs were readily observed in the proximal tibial sections of both 5- and 10-monthold mice, although the 10-month-old mice exhibited far fewer Nestin ${ }^{+}$BMMSCs. The p-Rock2-, p-Jak1, pTyr216-Gsk3 $\beta, \quad p-\beta$-catenin-, and active $\beta$-catenin- 


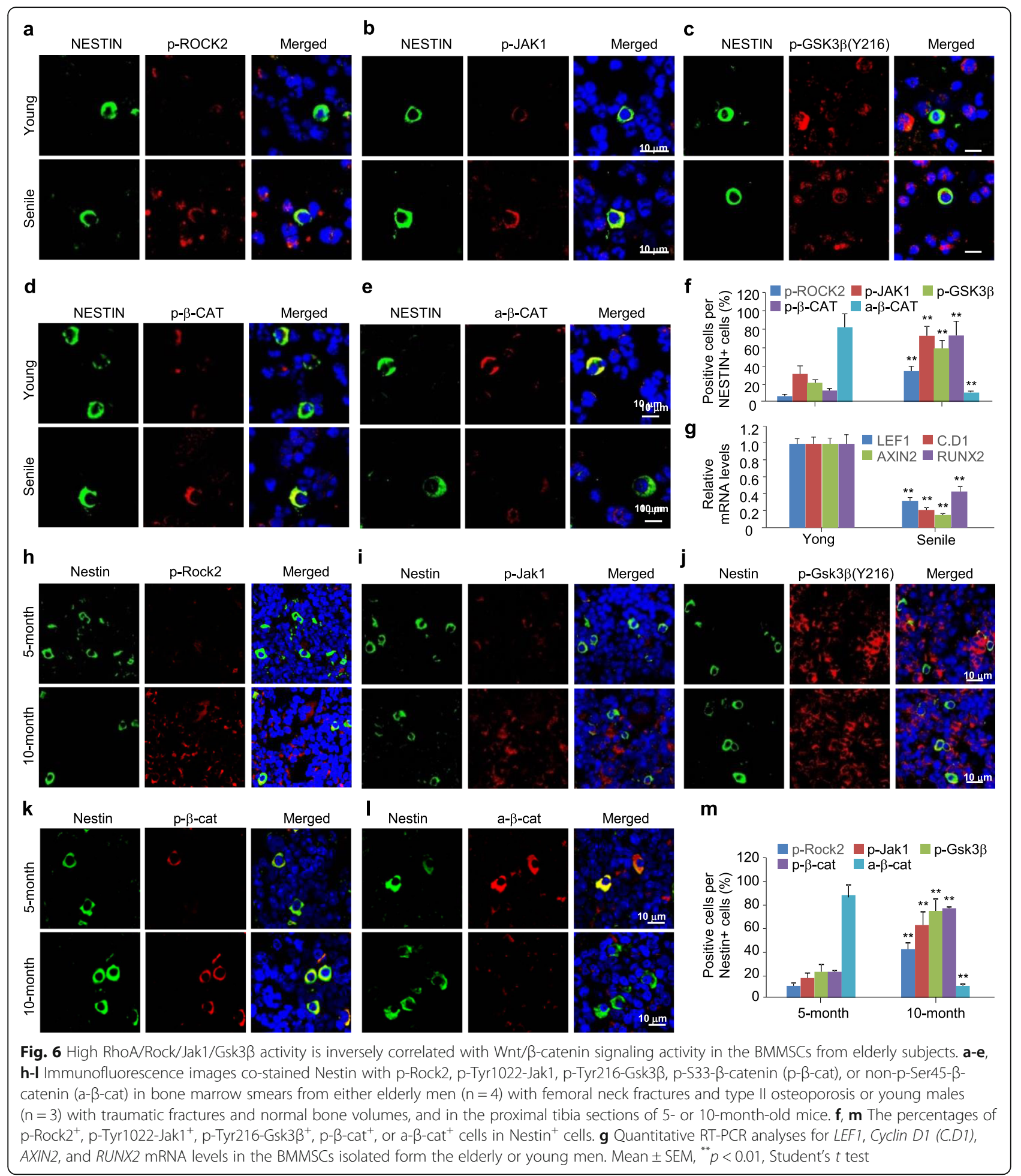

derived immunosignals were robustly detectable and the apparently overlapping signals between Nestin and other molecules were readily observed in the cytoplasm of the BMMSCs (Fig. 6h-l). The Nestin ${ }^{+}$BMMSCs in the tibial sections from 10-month-old mice showed significantly more $\mathrm{p}-\operatorname{Rock}_{2}{ }^{+}, \mathrm{p}-\mathrm{Jak} 1^{+}, \mathrm{p}-\mathrm{Tyr} 216-\mathrm{Gsk} 3 \beta^{+}$or $\mathrm{p}-\beta$ - catenin $^{+}$cells but less active $\beta$-catenin ${ }^{+}$cells when compared with the BMMSCs from the 5-month-old mice (Fig. 6h-m). Thus, RhoA/Rock activation was closely correlated with the activation of Jak and Tyr216-Gsk3 $\beta$ and inversely correlated with the inactivation of Wnt/ $\beta$ catenin signaling in the BMMSCs from elderly men. 
FZD receptors and Dkk1 coordinate RhoA/Rock activation in the regulation of $\beta$-catenin signaling in BMMSCs

We compared the $\beta$-catenin signaling and osteoblastogenic activities of BMMSCs from young and elderly mice in response to Wnt3a by isolating and culturing the BMMSCs from 2- and 8-month-old mice and determining the mRNA levels of $\beta$-catenin signaling targets, including Lef1, Axin2, and Cyclin D1, and of osteoblastogenic markers, including $A l p, B s p$, and Runx2. BMMSCs from two-month-old mice expressed significantly higher levels of these mRNAs than was observed in the 8month-old BMMSCs with or without the addition of Wnt3a. However, the mRNA levels were increased more robustly in response to Wnt3a stimulation in the BMMSCs from 2-month-old mice than from 8-monthold mice (Fig. 7a, b). Thus, the BMMSCs from elderly animals have a relatively low capacity to mediate the Wnt/ $\beta$-catenin signaling and osteoblastogenesis.

At present, 19 Wnt ligands and 10 FZD receptors have been identified, and the interactions between these ligands and receptors vary in a developmental and tissue-specific manner, leading to activation of $\beta$ catenin-dependent and/or $\beta$-catenin-independent Wnt signaling (Voloshanenko et al. 2017; DeBruine et al.
2017). We addressed whether the low activity of Wnt/ $\beta$ catenin signaling in the BMMSCs of the elderly mice results from the alterations in Wnt ligands and/or FZD receptors by examining the mRNA levels of Wnt3a, $W n t 5 a$, and $W n t 10 b$ (the representatives of Wnt ligands) and of FZD1 10. BMMSCs from 8-month-old mice displayed almost the same mRNA levels of Wnt3a, Wnt5a, and Wnt10 as were found in BMMSCs from 2-monthold mice (Fig. 7c), whereas the BMMSCs from the old mice showed significantly lower mRNA levels of FZD1, 4 , and 7 or higher levels of FZD3, 6, 8, and 9 (Fig. 7d). FZD2, 5, 7, and 9 participate in both the $\beta$-catenindependent and $\beta$-catenin-independent Wnt signaling, whereas FZD3, 6, and 8 are predominantly involved in the $\beta$-catenin-independent Wnt signaling, and FZD1, 4, and 9 mainly mediate $\beta$-catenin-dependent Wnt signaling (Voloshanenko et al. 2017; DeBruine et al. 2017). Thus, the down-regulation of FZD1, 4, and 7 expression and the upregulation of FZD3, 6, and 8 expression may lead to the aberrant activation of RhoA/Rock and the low responsiveness of $\mathrm{Wnt} / \beta$-catenin signaling in the BMMSCs from the old mice (Fig. 7h).

Wnt3a, which is capable of activating both the "canonical" and "non-canonical" Wnt signaling (Tu et al. 2007;

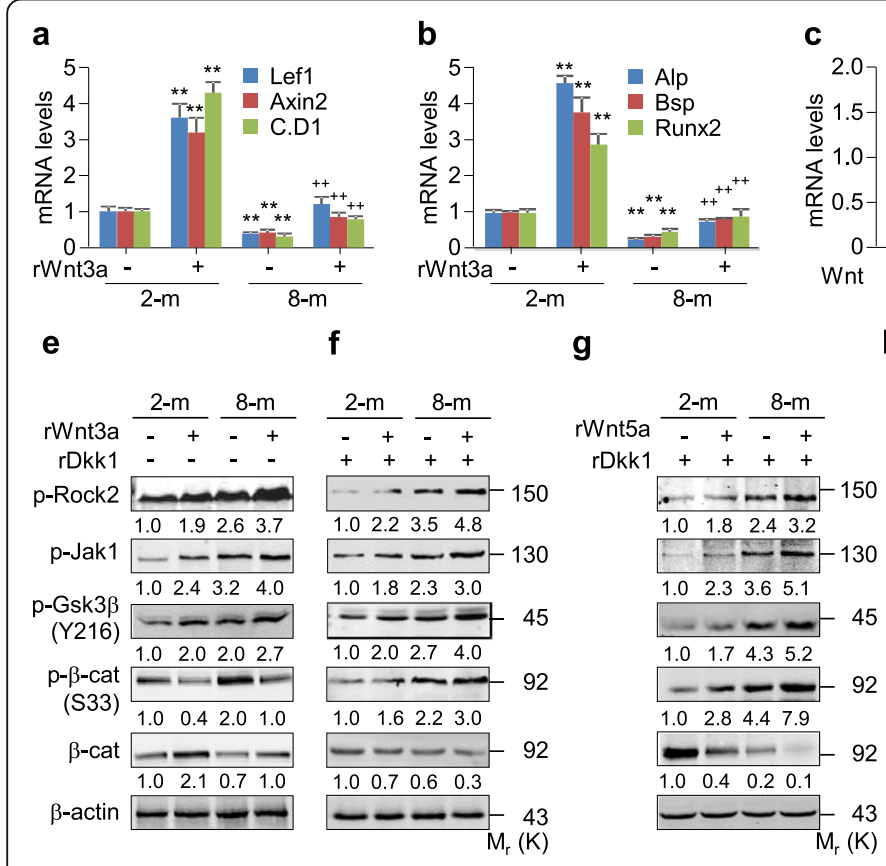

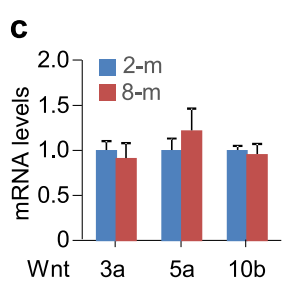

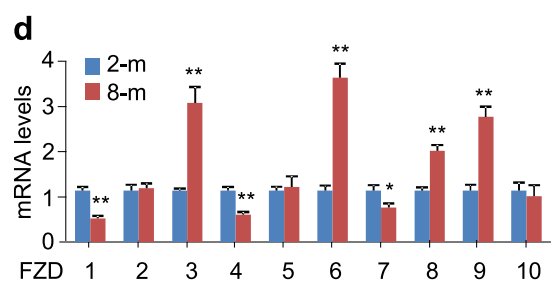

h

Fig. 7 FZD receptors and Dkk1 coordinate RhoA/Rock activation to destabilize $\beta$-catenin in the BMMSCs from elderly mice. a-d Quantitative RTPCR analyses for the indicated mRNA levels of BMMSCs isolated form 2- or 8-month-old mice and treated with or without rWnt3a at $100 \mathrm{ng} / \mathrm{ml}$ for $48 \mathrm{~h}$. Mean \pm SD, ${ }^{* *} p<0.01, \mathrm{n}=6$, Tukey-Kramer multiple comparisons test. e-g Western blotting analyses in the BMMSCs isolated form 2- or 8month-old mice and treated with or without rWnt3a/rWnt5a at $100 \mathrm{ng} / \mathrm{ml}$ in the presence or absence of rDkk1 at $100 \mathrm{ng} / \mathrm{ml}$ for $3 \mathrm{~h}$. $\mathbf{h}$ An integrated working model of $\beta$-catenin signaling mediated by RhoA/Rock in the regulation of aging-associated bone loss. In the BMMSCs from young subjects, APC/Axin/Gsk3 $\beta$ signaling mediated by FZD1, FZD4, and FZD7 overwhelms the RhoA/Rock/Jak/Gsk3 $\beta$ signaling mediated by FZD3, FZD6, and FZD8 to stabilize $\beta$-catenin and, in turn, enhance bone formation. However, in the BMMSCs from elderly subjects, the activation of RhoA/Rock/Jak/Gsk3 $\beta$ signaling mediated by FZD3, FZD6, and FZD8, in combination with the inactivation of APC/Axin/Gsk3 $\beta$ signaling mediated by Dkk1, Sost, and FZD1, FZD4, and FZD7 results in the destabilization of $\beta$-catenin and the subsequent attenuation of bone formation 
$\mathrm{Qu}$ et al. 2013), decreased the p-S33- $\beta$-catenin and increased the $\beta$-catenin levels more robustly in the BMMSCs from young mice than from the old mice, though Wnt3a treatment consistently increased the pRock2, p-Jak1, and p-Tyr216-Gsk3 $\beta$ in BMMSCs from mice at both ages (Fig. 7e). Thus, Wnt3a/FZDs/RhoA/ Rock/Jak-mediated Gsk3 $\beta$ activation counteracts the Wnt3a/FZDs/APC/Axin-mediated Gsk3 $\beta$ inactivation, resulting in the low responsiveness of $\mathrm{Wnt} / \beta$-catenin signaling in the BMMSCs from the old mice (Fig. 7h). These findings are consistent with the notion that "noncanonical Wnt signaling" inhibits "canonical Wnt signaling" (Nemeth et al. 2007; Yuzugullu et al. 2009).

The high circulating levels of Dkk1 and Sost cause a significantly low activity of $\mathrm{Wnt} / \beta$-catenin signaling in BMMSCs of patients with osteoporosis or age-associated bone loss (Coulson et al. 2017; Hampson et al. 2013). We explored the possibility that Dkk1 also functions as a molecular switch between the APC/Axin/Gsk3 $\beta$ signaling and the RhoA/Rock/Jak/Gsk3 $\beta$ signaling in controlling the $\beta$-catenin levels by western blot analyses. Unexpectedly, in the presence of Dkk1, Wnt3a treatment consistently increased the levels of p-Rock2, p-Jak1, p-Tyr216-Gsk3 $\beta$, and p-S33- $\beta$-catenin but decreased the level of $\beta$-catenin in BMMSCs from mice of both ages (Fig. 7f). This suggests that Dkk1 inactivates Wnt3a/FZDs/APC/Axin/Gsk3 $\beta$ signaling and, in turn, activates Wnt3a/FZDs/RhoA/Rock/Jak/Gsk3 $\beta$ signaling, resulting in the $\beta$-catenin destabilization and bone loss (Fig. 7h).

We further addressed the role of Dkk1 by treating the BMMSCs with a combination of Dkk1 and Wnt5a, which is considered as a "non-canonical" Wnt ligand and is capable of activating RhoA/Rock (Li et al. 2019; Uehara et al. 2017). Wnt5a behaved essentially the same as Wnt3a in the presence of Dkk1, as it robustly increased the levels of p-Rock2, p-Jak1, p-Tyr216-Gsk3 $\beta$, and $\mathrm{p}$-S33- $\beta$-catenin and consequently destabilizes $\beta$ catenin (Fig. 7g). Thus, FZDs/APC/Axin-mediated Gsk3 $\beta$ inactivation surpasses the FZDs/RhoA/Rock/Jakmediated Gsk3 $\beta$ activation, resulting in the $\beta$-catenin stabilization and bone formation in the BMMSCs from young mice. By contrast, FZDs/RhoA/Rock/Jak-mediated Gsk3 $\beta$ activation surpasses the FZDs/APC/Axin-mediated Gsk3 $\beta$ inactivation, resulting in the $\beta$-catenin destabilization and bone loss in the BMMSCs from old mice (Fig. 7h).

\section{Pharmacological inhibition of Rock antagonizes aging- associated bone loss by activating $\mathrm{Wnt} / \beta$-catenin signaling}

We examined whether the inhibition of RhoA/Rock is sufficient to activate $\mathrm{Wnt} / \beta$-catenin signaling and in turn enhance the bone volume in male mice with aging- associated bone loss by assessing the pharmacological effectiveness of Fasudil, a Rock2 inhibitor, as a preventative of this bone loss. Eight-month-old male wild-type mice were orally administered with $100 \mathrm{mg} / \mathrm{kg}$ of Fasudil once daily for 2 months, and then assayed for bone volume and activity of $W n t / \beta$-catenin signaling. At 10 months of age, the mice exhibited a significantly low trabecular bone mass, whereas Fasudil robustly preserved the mass of cancellous bone, as revealed by morphological and histological analyses (Fig. 8a, c). Threedimensional reconstruction of the proximal tibia using $\mu \mathrm{CT}$ confirmed that Fasudil treatment at $100 \mathrm{mg} / \mathrm{kg}$ resulted in a significant increase in BV/TV, Tb.N, and $\mathrm{Tb} . \mathrm{Th}$, but a robust decrease in Tb.Sp, when compared to mice administrated the vehicle (Fig. 8b). H\&E staining of longitudinal sections of the proximal tibiae consistently revealed that Fasudil treatment increased the Ob.N/BS and Ob.S/BS by 2.3- and 2.0-fold, respectively (Fig. 8c, d). By contrast, Trap-stained sections indicated that Fasudil treatment decreased the osteoclast characteristics, including Oc.N/BS and Oc.S/BS, by $41 \%$ and $45 \%$, respectively, compared to the vehicle treatment (Fig. 8e, f).

Consistent with the increase in bone volume, Fasudil treatment significantly increased the number of $\mathrm{Alp}^{+}$stained osteoblasts observed in horizontal sections of lumbar (Fig. S10A, B, D), whereas analyses of mRNA levels of osteoblast markers in femurs revealed that Fasudil treatment significantly increased the mRNA levels of Alp, Bsp, and Runx2 (Fig. 8g). Analyses of Lef1, Cyclin $D 1$, and Axin2 mRNA levels in femurs indicated that Fasudil treatment also robustly increased the expression of these Wnt/ß-catenin signaling targets (Fig. 8h), whereas immunostaining of Lef1 in horizontal sections of lumbar indicated that Fasudil treatment significantly increased the number of Lef $1^{+}$-stained cells (Fig. S10A, C, E). Moreover, dynamic bone histomorphometry with calcein labeling indicated that Fasudil treatment increased the BFR/BS, MAR, and dLS/BS by $118 \%, 120 \%$, and $116 \%$, respectively (Fig. $8 \mathrm{i}, \mathrm{j}$ ). Serological analysis of bone metabolism markers indicated Fasudil treatment increased the levels of bone formation markers P1NP and $\mathrm{OCN}$ by $52 \%$ and $62 \%$, respectively, but decreased the levels of bone resorption markers CTX and TRAP by $37 \%$ and $42 \%$, respectively, compared to the vehicle treatment (Fig. S10F-I). Overall, these data reveal that pharmacological inhibition of RhoA/Rock prevents the aging-associated bone loss, possibly by activating $\mathrm{Wnt} / \beta$ catenin signaling.

\section{Discussion}

During Wnt/ $\beta$-catenin signaling, Wnt3a on the one hand suppresses APC/Axin/Gsk3 $\beta$ to stabilize $\beta$-catenin (Wu et al. 2008), but on the other hand activates RhoA/ 


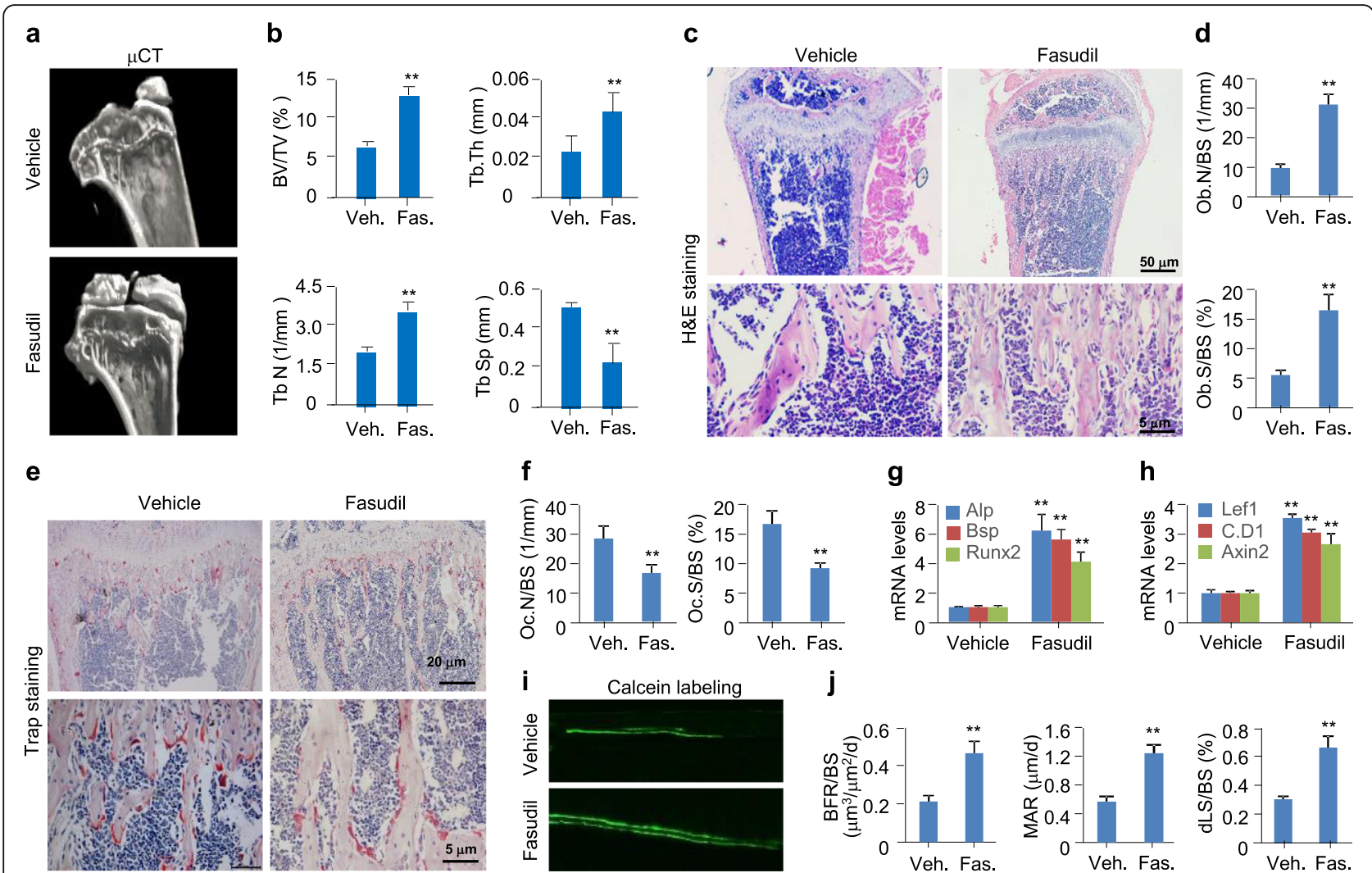

Fig. 8 Pharmacological inhibition of Rock by Fasudil antagonizes aging-associated bone loss. 8-month-old male mice were orally administrated with vehicle (Veh.) or Fasudil (Fas.) at $100 \mathrm{mg} / \mathrm{kg}$ once daily for 2 months. Tibias were harvested for the following analyses. a, b Representative $\mu C T$ images and parameters including BV/TV, Tb.Th, Tb.N, and Tb.Sp for the proximal tibias. $\mathbf{c}$, d Representative images of H\&E staining and quantification of Ob.N/BS and Ob.S/BS for the proximal tibia sections. e, $\mathbf{f}$ Representative images of Trap staining and quantification of Oc.N/BS and Oc.S/BS for the proximal tibia sections. $\mathbf{g}, \mathbf{h}$ qPCR analyses for Alp, Bsp, Runx2, Lef1, Cyclin D1 (C.D1), and Axin2 mRNA levels in femurs. (i, j) Dynamic bone histomorphometry and quantification for BFR/BS, MAR, and dLS/BS. Mean \pm SEM, ${ }^{*} p<0.05,{ }^{* *} p<0.01, \mathrm{n}=8$, Tukey-Kramer multiple comparisons test

Rock to destabilize $\beta$-catenin by activating Jak and Gsk3 $\beta$ (Fig. 7h). Thus, RhoA/Rock activation constitutes a negative regulatory mechanism that allows fine-tuning the amplitude of $\beta$-catenin signaling.

The limb phenotypes resulting from RhoA loss-offunction or gain-of-function in the AER can also arise in the presence, rather than the absence, of the $D k k 1$ transgene. This suggests that RhoA loss-of function or gainof-function in the wildtype background underscores both the robustness of the $\mathrm{Wnt} / \beta$-catenin signaling system and the sensitivity of the AER to a threshold level of Wnt signaling. Thus, under physiological conditions, the $\beta$-catenin stabilization mediated by APC/Axin/GSK3 $\beta$ signaling overwhelms the destabilization mediated by RhoA/Rock/Jak/Gsk3 $\beta$ signaling to regulate the limb outgrowth. By contrast, RhoA loss-of-function or gainof-function in pre-osteoblasts is sufficient to cause the bone phenotypes, and aberrant activation of RhoA/Rock in aging-associated bone loss causes the inactivation of $\beta$-catenin in the BMMSCs from elderly subjects. Thus, under pathological conditions, such as type II osteoporosis, the $\beta$-catenin destabilization mediated by RhoA/Rock/Jak/Gsk3 $\beta$ signaling overwhelms the $\beta$ catenin stabilization mediated by APC/Axin/GSK3 $\beta$ signaling to regulate the bone volume.

Fasudil, an inhibitor of Rock2, has been reported to induce osteoblastic differentiation by promoting BMP2 expression (Kanazawa et al. 2010). Here, we present an alternative mechanism wherein Fasudil antagonizes aging-associated bone loss. Both the previous and our present studies demonstrate a therapeutic potential for Fasudil as a preventative treatment for osteoporosis (Shimokawa and Takeshita 2005). Likewise, AZD2858, a Gsk3 inhibitor, significantly increases the bone mass in rats by upregulating the $\beta$-catenin levels (Marsell et al. 2012), further validating this inhibitor in the osteoporosis therapy by targeting the ROCK/JAK/GSK3 $\beta$ signaling.

Although RhoA-mediated Wnt/ $\beta$-catenin signaling has been identified by the previous works (Ordóñez-Morán et al. 2008; Rodrigues et al. 2014; Miyagi et al. 2015; Kim et al. 2017; Rossol-Allison et al. 2009) and in the present 
study, a role of RhoA-mediated $\beta$-catenin-independent Wnt signaling has also been established for the osteogenic differentiation in response to mechanical stimulation (Shi et al. 2012; Li et al. 2015; Li et al. 2019). In addition, RhoA mediates Wnt5a/Ror2 signaling to improve the actin ring formation in osteoclasts and to facilitate bone resorption (Uehara et al. 2017). Thus, these findings support the notion that the actual role of RhoA/Rock in $\beta$-catenin-dependent or $\beta$-cateninindependent Wnt signaling depends on the duration, degree and the cellular and tissue context.

How GSK3 $\beta$ is modulated and fine-tuned by Wnt to stabilize $\beta$-catenin remains a fundamental question in the field. We suggest that RhoA/Rock activates Jak by directly phosphorylating Jak at Tyr216, consistent with the Jak activation by Wnt3a (Fragoso et al. 2012; Hao et al. 2006). Generally, Jak is recruited from the cytoplasm to bind and activate cytokine receptors upon the cytokine stimulation. Here, we suggest that cytoplasmic Jak is recruited into the protein complex consisting of Rock and Gsk $3 \beta$ during Wnt signaling.

One recent study has indicated that Rock1 physically interacts with and activates Jak2 in response to leptin (Huang et al. 2013). However, the detailed mechanism by which RhoA/Rock regulates Jak during Wnt signaling is unknown and warrants further study. Although emerging evidences emphasizes the importance of Jak/Stat pathways in bone development and metabolism ( $\mathrm{Li}$ 2013), the findings presented here suggest that Jak activation destabilizes $\beta$-catenin and prevents the maintenance of bone volume, consistent with the finding that constitutive Jak2 activation in mesenchymal cells promotes adipogenesis and reduces osteogenesis in the bone marrow (Yue et al. 2016).

Jak inhibitors are approved as treatments for rheumatoid arthritis, however, the role of Jak/Stat inhibition in bone homeostasis is not fully understood. Ruxolitinib, an inhibitor of Jak/Stat, decreases osteoblastic differentiation of hMSCs in vitro and reduces ectopic bone formation in vivo (AlMuraikhi et al. 2018). However, these findings appear to be at odds with our present finding that P6, a pan-JAK inhibitor, stabilizes $\beta$-catenin and promotes osteoblast differentiation. They also differ from a recent study that showed that the Jak inhibitors tofacitinib and baricitinib significantly increase osteoblast function and bone mass by stabilizing $\beta$-catenin in vivo, with no direct effects on osteoclasts in vitro (Adam et al. 2020). These discrepancies could be explained by the fact that the interactions among JAKs and STATs occur in a developmental and tissue-specific manner and that JAK/STAT signaling pathways have diverse effects in different cell contexts (Murray 2007; Gadina et al. 2018).
In conclusion, we identify RhoA/Rock/Jak/ Gsk3 3 (Tyr216) as a hitherto uncharacterized signaling cascade that acts primarily to destabilize $\beta$-catenin. Thus, this study highlights a precise regulation of Wnt signaling and provides an additional mechanism for regulation of limb development, while also identifying a potential new therapeutic target for aging-associated bone loss.

\section{Supplementary Information}

The online version contains supplementary material available at https://doi. org/10.1186/s13619-020-00071-3.

\section{Additional file 1.}

Additional file 2.

\section{Abbreviations}

BMMSCs: Bone marrow-derived mesenchymal stromal cells; FZD: Frizzled; Lrp5/6: Low-density lipoprotein receptor-related protein 5 or 6;

Dvl: Dishevelled; Gsk3ß: Glycogen synthase kinase-3ß; APC: Adenomatous polyposis coli; Lef1: Lymphoid enhancer-binding factor 1; (Tcf): T cell factors; Dkk1: Dickkopf 1; PCP: Planar cell polarity; CE: Convergent extension; JNK2: $C^{-}$ Jun N-terminal kinase 2; Rock: Rho-kinase; Jak: Janus kinase; $\beta$-catenin: $\beta$-cat; PMCOBs: Primary murine calvarial osteoblasts; dnRhoA: Dominant negative form of RhoA; caRhoA: Constitutively active form of RhoA; Col1: Collagen1a1; caRock2: Constitutively active form of Rock2; Alp: Alkaline phosphatase; Akt: Protein kinase B; WT: Wild type; P6: Pyridone 6; AER: Apical ectodermal ridge; Fgf8: Fibroblast growth factor 8; Bmp4: Bone morphogenetic protein 4; $\mu \mathrm{CT}$ : Microcomputer tomography; BV/TV: Bone volume/trabecular volume; Tb.Th: Trabecular thickness; Tb.N: Trabecular number; Tb.Sp: Trabecular separation; Ob.N/BS: Osteoblast number/bone surface; Ob.S/BS: Osteoblast surface/bone surface; Trap: Tartrate-resistant acid phosphatase; Oc.N/ BS: Osteoclast number/bone surface; Oc.S/BS: Osteoclast surface/bone surface; BFR/BS: Bone formation rate/bone surface; MAR: Mineral apposition rate; dLS/BS: Double-labeled surface/bone surface; Bsp: Bone sialoprotein; P1NP: Procollagen type $1 \mathrm{~N}$-terminal propeptide; OCN: Osteocalcin; CTX: Carboxyterminal collagen cross-links; STR: Short tandem repeat; FBS: Fetal bovine serum; GAPDH: Glyceraldehyde-3-phosphate dehydrogenase; $\beta$-GP: $\beta$-glycerophosphate.

\section{Acknowledgements}

We are indebted to Drs. Xi He, Christof Niehrs, and Walter Koch for their reagents and to Dr. kazuto Kobayashi for CAT-dnRhoA founders.

\section{Authors' contributions}

L-H.Z. and X.W. were the main contributors in the conception, design, and interpretation of the data. W.S., C.X., J.W.,Z.Y., Y.G., and L.M. performed the in vitro experiments and data analysis. W.S., C.X., Y.G., J.W., Q.R., X.H., and M.Q. performed the in vivo experiments, whole-mount skeleton preparation, whole-mount in situ hybridization, and histological examination. C.T., X.J., and M.H. were responsible for image analysis. W.S., L-H.Z., and X.W. wrote this manuscript.

\section{Funding}

This work was supported by 973 Program (No. 2018YFC1004404) and National Natural Science Foundation of China (Nos. 31071292, 31271561, 31571493, 81741043, 31871395, and 31801207).

\section{Availability of data and materials}

Dataset described in this work can be downloaded from the supplementary Figs. S1-10 and Tables S1-3 from the journal website. Materials request should be addressed to the corresponding author.

\section{Ethics approval and consent to participate}

All animal experiments were conducted according to the institutional guidelines for laboratory animals, and the protocols were approved by the Zhejiang University Institutional Animal Care and Use Committee. For all 
human studies, written informed consent was received from participants with no financial incentive provided, and samples were obtained in accordance with the standard protocols of Ethics Committee of Zhejiang University School of Medicine.

\section{Competing interests}

The authors declare no competing interests.

\section{Author details}

'Department of Pharmacology, Zhejiang University School of Medicine, 866 Yuhangtang Road, Hangzhou 310058, China. ²Department of Biology and Genetics, University of North Carolina-Chapel Hill, Chapel Hill, NC 27599, USA. ${ }^{3}$ Department of Orthopeadic Surgery of Sir Run Run Shaw Hospital, Zhejiang University School of Medicine, Hangzhou 310016, China. ${ }^{4}$ Department of Pharmacology, Zhejiang University City College, 51 Huzhou Street, Hangzhou 310015, China. ${ }^{5}$ Translational Research Program in Pediatric Orthopaedics, The Children's Hospital of Philadelphia, Philadelphia, PA 19104, USA.

\section{Received: 22 July 2020 Accepted: 25 November 2020}

\section{Published online: 03 March 2021}

\section{References}

Adam S, Simon N, Steffen U, Andes FT, Scholtysek C, Müller DIH, et al. JAK inhibition increases bone mass in steady-state conditions and ameliorates pathological bone loss by stimulating osteoblast function. Sci Transl Med. 2020;12:eaay4447

AlMuraikhi N, Ali D, Alshanwani A, Vishnubalaji R, Manikandan M, Atteya M, et al. Stem cell library screen identified ruxolitinib as regulator of osteoblastic differentiation of human skeletal stem cells. Stem Cell Res Ther. 2018:9:319.

Bafico A, Liu G, Yaniv A, Gazit A, Aaronson SA. Novel mechanism of Wnt signalling inhibition mediated by Dickkopf-1 interaction with LRP6/arrow. Nat Cell Biol. 2001;3:683-6.

Barrow JR, Thomas KR, Boussadia-Zahui O, Moore R, Kemler R, Capecchi MR, et al. Ectodermal Wnt3/beta-catenin signaling is required for the establishment and maintenance of the apical ectodermal ridge. Genes Dev. 2003;17:394-409.

Bishop AL, Hall A. Rho GTPases and their effector proteins. Biochem J. 2000;348: 241-55.

Canalis E, Giustina A, Bilezikian JP. Mechanisms of anabolic therapies for osteoporosis. N Engl J Med. 2007;357:905-16.

Chen $\mathrm{H}$, Ji X, Hu X, Chen L, Lv H, Xu C, et al. Inhibition of heat shock protein 90 rescues glucocorticoid-induced bone loss through enhancing bone formation. J Steroid Biochem Mol Biol. 2017;171:236-46.

Coulson J, Bagley L, Barnouin Y, Bradburn S, Butler-Browne G, Gapeyeva H, et al. Circulating levels of dickkopf-1, osteoprotegerin and sclerostin are higher in old compared with young men and women and positively associated with whole-body bone mineral density in older adults. Osteoporos Int. 2017;28: 2683-9.

DeBruine ZJ, Xu HE, Melcher K. Assembly and architecture of the Wnt/B-catenin signalosome at the membrane. Br J Pharmacol. 2017;174:4564-74.

Dempster DW, Compston JE, Drezner MK, Glorieux FH, Kanis JA, Malluche H, et al. Standardized nomenclature, symbols, and units for bone histomorphometry: a 2012 update of the report of the ASBMR Histomorphometry nomenclature committee. J Bone Miner Res. 2013;28:2-17.

Fanto M, Weber U, Strutt DI, Mlodzik M. Nuclear signaling by Rac and rho GTPases is required in the establishment of epithelial planar polarity in the drosophila eye. Curr Biol. 2000;10:979-88.

Fragoso MA, Patel AK, Nakamura RE, Yi H, Surapaneni K, Hackam AS. The Wnt/Bcatenin pathway cross-talks with STAT3 signaling to regulate survival of retinal pigment epithelium cells. PLoS One. 2012;7:e46892.

Gadina M, Johnson C, Schwartz D, Bonelli M, Hasni S, Kanno Y, et al. Translational and clinical advances in JAK-STAT biology: the present and future of jakinibs. J Leukoc Biol. 2018;104:499-514.

Gong Y, Xu CY, Wang JR, Hu XH, Hong D, Ji X, et al. Inhibition of phosphodiesterase 5 reduces bone mass by suppression of canonical Wnt signaling. Cell Death Dis. 2014;5:e1544.

Hampson G, Edwards S, Conroy S, Blake GM, Fogelman I, Frost ML. The relationship between inhibitors of the Wnt signalling pathway (Dickkopf1 (DKK1) and sclerostin), bone mineral density, vascular calcification and arterial stiffness in post-menopausal women. Bone. 2013;56:42-7.
Hao J, Li T, Qi X, Zhao D, Zhao G. Wnt/beta-catenin pathway up-regulates Stat3 and converges on LIF to prevent differentiation of mouse embryonic stem cells. Dev Biol. 2006;290:81-91.

Hu X, Ji X, Yang M, Fan S, Wang J, Lu M, et al. Cdc42 is essential for both articular cartilage degeneration and subchondral bone deterioration in experimental osteoarthritis. J Bone Miner Res. 2018;33:945-58.

Huang $\mathrm{H}$, Kong D, Byun KH, Ye C, Koda S, Lee DH, et al. Rho-kinase regulates energy balance by targeting hypothalamic leptin receptor signaling. Nat Neurosci. 2013;15:1391-408.

Huelsken J, Birchmeier W. New aspects of Wnt signaling pathways in higher vertebrates. Curr Opin Genet Dev. 2001;11:547-53.

Jackson EL, Willis N, Mercer K, Bronson RT, Crowley D, Montoya R, et al. Analysis of lung tumor initiation and progression using conditional expression of oncogenic K-ras. Genes Dev. 2001;15:3243-8.

Jing H, Su X, Gao B, Shuai Y, Chen J, Deng Z, et al. Epigenetic inhibition of Wnt pathway suppresses osteogenic differentiation of BMSCs during osteoporosis. Cell Death Dis. 2018;9:176.

Kanazawa I, Yamaguchi T, Yano S, Yamauchi M, Sugimoto T. Activation of AMP kinase and inhibition of rho kinase induce the mineralization of osteoblastic MC3T3-E1 cells through endothelial NOS and BMP-2 expression. Am J Physio Endocrinol Metab. 2009;296:E139-46.

Kanazawa I, Yamaguchi T, Yano S, Yamauchi M, Sugimoto T. Fasudil hydrochloride induces osteoblastic differentiation of stromal cell lines, C3H10T1/2 and ST2, via bone morphogenetic protein-2 expression. Endocr J. 2010;57:415-21.

Karner CM, Long F. Wnt signaling and cellular metabolism in osteoblasts. Cell Mol Life Sci. 2017;74:1649-57.

Kim JG, Kim MJ, Choi WJ, Moon MY, Kim HJ, Lee JY, et al. Wnt3A induces GSK-3ß phosphorylation and $\beta$-catenin accumulation through RhoA/ROCK. J Cell Physiol. 2017;232:1104-13

Kobayashi K, Takahashi M, Matsushita N, Miyazaki J, Koike M, Yaginuma H, et al. Survival of developing motor neurons mediated by rho GTPase signaling pathway through rho-kinase. J Neurosci. 2004:24:3480-8.

Li J. JAK-STAT and bone metabolism. JAKSTAT. 2013;2:e23930.

Li J, Hu C, Han L, Liu L, Jing W, Tang W, et al. MiR-154-5p regulates osteogenic differentiation of adipose-derived mesenchymal stem cells under tensile stress through the Wnt/PCP pathway by targeting Wnt11. Bone. 2015;78:130-41.

Li R, Lin S, Zhu M, Deng Y, Chen X, Wei K, et al. Synthetic presentation of noncanonical Wnt5a motif promotes mechanosensing-dependent differentiation of stem cells and regeneration. Sci Adv. 2019;5:eaaw3896.

Ma Y, Zhang X, Shen B, Lu Y, Chen W, Ma J, et al. Generating rats with conditional alleles using CRISPR/Cas9. Cell Res. 2014;24:122-5.

Manolagas SC, Almeida M. Gone with the Wnts: beta-catenin, T-cell factor, forkhead box $\mathrm{O}$, and oxidative stress in age-dependent diseases of bone lipid, and glucose metabolism. Mol Endocrinol. 2007;21:2605-14.

Marsell R, Sisask G, Nilsson Y, Sundgren-Andersson AK, Andersson U, Larsson S, et al. GSK-3 inhibition by an orally active small molecule increases bone mass in rats. Bone. 2012:50:619-27.

Miyagi A, Negishi T, Yamamoto TS, Ueno N. G protein-coupled receptors Flop1 and Flop2 inhibit Wnt/ß-catenin signaling and are essential for head formation in Xenopus. Dev Biol. 2015;407:131-44.

Müller P, Kuttenkeuler D, Gesellchen V, Zeidler MP, Boutros M. Identification of JAK/STAT signalling components by genome-wide RNA interference. Nature. 2005:436:871-5.

Murray PJ. The JAK-STAT signaling pathway: input and output integration. J Immunol. 2007;178:2623-9.

Nemeth MJ, Topol L, Anderson SM, Yang Y, Bodine DM. Wnt5a inhibits canonical Wnt signaling in hematopoietic stem cells and enhances repopulation. Proc Natl Acad Sci U S A. 2007;104:15436-41.

Ohnaka K, Shimoda S, Nawata H, Shimokawa H, Kaibuchi K, Iwamoto Y, et al. Pitavastatin enhanced BMP-2 and osteocalcin expression by inhibition of rho-associated kinase in human osteoblasts. Biochem Biophys Res Commun. 2001:287:337-42.

Ordóñez-Morán P, Larriba MJ, Pálmer HG, Valero RA, Barbáchano A, Duñach M, et al. RhoA-ROCK and p38MAPK-MSK1 mediate vitamin D effects on gene expression, phenotype, and Wnt pathway in colon cancer cells. J Cell Biol. 2008:183:697-710.

Orgaz JL, Pandya P, Dalmeida R, Karagiannis P, Sanchez-Laorden B, Viros A, et al. Diverse matrix metalloproteinase functions regulate cancer amoeboid migration. Nat Commun. 2014;5:4255.

Papakonstanti EA, Ridley AJ, Vanhaesebroeck B. The p110delta isoform of PI3kinase negatively controls RhoA and PTEN. EMBO J. 2007;26:3050-61. 
Peng L, Li Y, Shusterman K, Kuehl M, Gibson CW. Wnt-RhoA signaling is involved in dental enamel development. Eur J Oral Sci. 2011;119(Suppl 1):41-9.

Qu F, Wang J, Xu N, Liu C, Li S, Wang N, et al. WNT3A modulates chondrogenesis via canonical and non-canonical WNT pathways in MSCs. Front Biosci (Landmark Ed). 2013;18:493-503.

Rodriques P, Macaya I, Bazzocco S, Mazzolini R, Andretta E, Dopeso H, et al. RHOA inactivation enhances Wnt signalling and promotes colorectal cancer. Nat Commun. 2014;5:5458.

Rossol-Allison J, Stemmle LN, Swenson-Fields Kl, Kelly P, Fields PE, McCall SJ, et al. Rho GTPase activity modulates Wnt3a/beta-catenin signaling. Cell Signal. 2009:21:1559-68

Shi Y, Fu Y, Tong W, Geng Y, Lui PP, Tang T, et al. Uniaxial mechanical tension promoted osteogenic differentiation of rat tendon-derived stem cells (rTDSCs) via the Wnt5a-RhoA pathway. J Cell Biochem. 2012;113:3133-42.

Shimokawa $\mathrm{H}$, Takeshita A. Rho-kinase is an important therapeutic target in cardiovascular medicine. Arterioscler Thromb Vasc Biol. 2005;25:1767-75.

Shusterman K, Gibson CW, Li Y, Healey M, Peng L. Wnt-RhoA signaling pathways in fluoride-treated ameloblast-lineage cells. Cells Tissues Organs. 2014;199: 159-68.

Strutt DI, Weber U, Mlodzik M. The role of RhoA in tissue polarity and frizzled signalling. Nature. 1997;387:292-5.

Sun X, Lewandoski M, Meyers EN, Liu YH, Maxson RE Jr, Martin GR. Conditional inactivation of Fgf4 reveals complexity of signaling during limb bud development. Nat Genet. 2000;25:83-6.

ter Haar E, Coll JT, Austen DA, Hsiao HM, Swenson L, Jain J. Structure of GSK3beta reveals a primed phosphorylation mechanism. Nat Struct Biol. 2001;8:593-606.

Tu X, Joeng KS, Nakayama Kl, Nakayama K, Rajagopal J, Carroll TJ, et al. Noncanonical Wnt signaling through G protein-linked PKCdelta activation promotes bone formation. Dev Cell. 2007;12:113-27.

Uehara S, Udagawa N, Mukai H, Ishihara A, Maeda K, Yamashita T, et al. Protein kinase N3 promotes bone resorption by osteoclasts in response to Wnt5aRor2 signaling. Sci Signal. 2017;10:eaan0023.

van Amerongen R, Nusse R. Towards an integrated view of Wnt signaling in development. Development. 2009:136:3205-14.

Voloshanenko O, Gmach P, Winter J, Kranz D, Boutros M. Mapping of Wnt-frizzled interactions by multiplex CRISPR targeting of receptor gene families. FASEB $J$. 2017;31:4832-44

Wan Q, Cho E, Yokota H, Na S. Rac1 and Cdc42 GTPases regulate shear stressdriven $\beta$-catenin signaling in osteoblasts. Biochem Biophys Res Commun. 2013;433:502-7.

Wu D, Pan W. GSK3: a multifaceted kinase in Wnt signaling. Trends Biochem Sci. 2010;35:161-8

Wu X, Tu X, Joeng KS, Hilton MJ, Williams DA, Long F. Rac1 activation controls nuclear localization of beta-catenin during canonical Wnt signaling. Cell. 2008;133:340-53.

Xu C, Wu X, Lu M, Tang L, Yao H, Wang J, et al. Protein tyrosine phosphatase 11 acts through RhoA/ROCK to regulate eosinophil accumulation in the allergic airway. FASEB J. 2019;33:11706-20.

Yue R, Zhou BO, Shimada IS, Zhao Z, Morrison SJ. Leptin receptor promotes Adipogenesis and reduces Osteogenesis by regulating Mesenchymal stromal cells in adult bone marrow. Cell Stem Cell. 2016;18:782-96.

Yuzugullu H, Benhaj K, Ozturk N, Senturk S, Celik E, Toylu A, et al. Canonical Wnt signaling is antagonized by noncanonical Wnt5a in hepatocellular carcinoma cells. Mol Cancer. 2009:8:90.

\section{Submit your manuscript to a SpringerOpen ${ }^{\circ}$ journal and benefit from:}

- Convenient online submission

- Rigorous peer review

- Open access: articles freely available online

- High visibility within the field

- Retaining the copyright to your article

Submit your next manuscript at $\boldsymbol{\nabla}$ springeropen.com 